\title{
Optimal Dispatch in a Balancing Market with Intermittent Renewable Generation
}

\author{
Priyanka Shinde, Student Member, IEEE, Mohammad Reza Hesamzadeh, Senior Member, IEEE, \\ Paresh Date, and Derek W. Bunn
}

\begin{abstract}
In this paper, three single-stage stochastic programs are proposed and compared for optimal dispatch by a System Operator (SO) into balancing markets (BM). The motivation for the models is to represent a possible requirement to undertake system balancing with increasing amounts of intermittent renewable generation. The proposed optimization models are reformulated as tractable Mixed Integer Linear Programs (MILPs) and these consider both fuel cost and intermittency cost of the generators, when the SO activates the up- or down-regulation bids. These three models are based on the main approaches seen in practice: dual-imbalance pricing, single imbalance pricing and single imbalance pricing with spot reversion. A scenariogeneration algorithm based on predictive conditional dynamic density distributions is also proposed. We perform a comparative analysis of these three proposed models in terms of how they help the SO to optimize their balancing market actions considering intermittent-renewable generators. The single imbalance pricing is found to be the most market efficient.
\end{abstract}

Index Terms-Balancing market, Imbalance settlement cost (ISC), Stochastic program, GAMLSS

\section{NOMENCLATURE}

The main notation is presented below for a quick reference. Additional symbols are introduced throughout the text.

Indices

$i$ generating unit

$\omega$ scenarios of power generation

\section{Parameters}

$g_{i, \omega}^{m} \quad$ metered output of generator $i$ in scenario $w$

$g_{i}^{s} \quad$ scheduled output of generator $i$

$g_{i}^{u p} \quad$ up-regulation quantity bid of generator $i$

$g_{i}^{d n} \quad$ down-regulation quantity bid of generator $i$

$\lambda_{i}^{u p} \quad$ up-regulation price bid of generator $i$

$\lambda_{i}^{d n} \quad$ down-regulation price bid of generator $i$

SIL System Imbalance Level

$\beta \quad$ CVaR parameter (specified probability level)

$\pi_{\omega} \quad$ Probability weight of scenario $\omega$

$\mathcal{G}$ Total number of generators

$\lambda^{\text {spot }}$ Spot price

$\mu \quad$ Mean of metered output of generators

Manuscript received June 5, 2019; revised October 23, 2019 and February 24, 2020; accepted July 26, 2020.

P. Shinde is with the School of Electrical Engineering and Computer Science, KTH Royal Institute of Technology, Sweden.

M.R. Hesamzadeh is with the School of Electrical Engineering and Computer Science, KTH Royal Institute of Technology, Sweden; Program on Energy and Sustainable Development (PESD), Stanford University and German Institute for Economic Research (DIW Berlin).

P. Date is with the College of Engineering, Design and Physical Sciences, Brunel University, United Kingdom.

D. Bunn is with the Decision Sciences, Management Science and Operations, London Business School, United Kingdom. $\sigma \quad$ Standard deviation of metered output of generators wind $_{t}$ Wind generation in MWh at time $t$

dem $_{t}$ Demand in MWh at time $t$

$h y d_{t}$ Hydropower in MWh at time $t$

$\Xi \quad$ Big-M parameter

$\epsilon \quad$ Constant used in linearizing 'If' condition

\section{Variables}

$\phi_{i}^{u p} \quad$ Binary variable for up-regulation bid of generator $i$

$\phi_{i}^{d n} \quad$ Binary variable for down-regulation bid of generator $i$

$\bar{\phi} \quad$ Binary variable for system up-regulation status

$\phi \quad$ Binary variable for system down-regulation status

$\bar{\lambda}_{r e g}^{d n}$ Down-regulation price

$\lambda_{\text {reg }}^{u p}$ Up-regulation price

$\delta_{i, \omega}^{m}$ Imbalance quantity of generator $i$ in scenario $w$

$\bar{\alpha}_{\beta} \quad$ Surplus generation for a given probability value $\beta$

$\alpha_{\beta} \quad$ Unmet level of demand for a given probability value $\beta$

$\Delta_{\omega} \quad$ Net deviation in scenario $\omega$

$\Delta_{\omega}^{-} \quad$ Net negative deviation in scenario $\omega$

$\Delta_{\omega}^{+} \quad$ Net positive deviation in scenario $\omega$

$b_{\omega}^{-} \quad$ Binary variable to linearize $\Delta_{\omega}^{-}$

$b_{\omega}^{+} \quad$ Binary variable to linearize $\Delta_{\omega}^{+}$

Variables used in DIP model for Big-M linearization

$I_{i, w}^{+} \quad$ Bilinear variable for positive deviation and down-regulation

$I_{i, w}^{-} \quad$ Bilinear variable for negative deviation and up-regulation

$s_{\omega} \quad$ Non-linear term for $\mathbb{E}$ (ISC) due to negative deviation

$c_{\omega} \quad$ Non-linear term for $\mathbb{E}$ (ISC) due to positive deviation

$I_{i w}^{u p} \quad$ Non-linear variable equal to product of $s_{\omega}$ and $\phi_{i}^{u p}$

$I_{i, w}^{d n} \quad$ Non-linear variable equal to product of $c_{\omega}$ and $\phi_{i}^{d n}$

$z_{\omega}^{d n} \quad$ Variable to linearize the product of $\phi_{i}^{d n}$ and $c_{\omega}$

$z_{\omega}^{u p} \quad$ Variable to linearize the product of $\phi_{i}^{u p}$ and $s_{\omega}$

$u_{\omega}^{-} \quad$ Non-linear variable for linearizing CVaR constraint for unmet demand

$u_{\omega}^{+} \quad$ Non-linear variable for linearizing CVaR constraint for surplus generation

$b_{\omega}^{1} \quad$ Binary variable to linearize Max operator for surplus generation

$b_{\omega}^{2} \quad$ Binary variable to linearize Max operator for unmet demand

Variables used in SIP-SR model for Big-M linearization $N_{i, \omega}^{u p} \quad$ Bilinear variable equal to product of $\phi_{i}^{u p}$ and $\Delta_{\omega}^{-}$

$N_{i, \omega}^{d n} \quad$ Bilinear variable equal to product of $\phi_{i}^{d n}$ and $\Delta_{\omega}^{+}$

$c_{\omega}^{u p} \quad$ Bilinear variable related to up-regulation cost

$c_{\omega}^{d n} \quad$ Bilinear variable related to down-regulation cost 
$q_{i \omega} \quad$ Binary variable to identify marginal down-regulating generator

$r_{i \omega} \quad$ Binary variable to identify marginal up-regulating generator

$\phi \quad$ Binary variable to indicate equal activated volumes for up- and down-regulation

$k_{i}^{u p} \quad$ Non-linear variable equal to product of $\phi_{i}^{u p}$ and $\phi$

$k_{i}^{d n} \quad$ Non-linear variable equal to product of $\phi_{i}^{d n}$ and $\phi$

$h^{+} \quad$ Bilinear variable equal to product of $\Delta_{\omega}^{+}$and $\phi$

$h^{-} \quad$ Bilinear variable equal to product of $\Delta_{\omega}^{-}$and $\phi$

$t_{\omega}^{u p} \quad$ Bilinear variable equal to product of $\Delta_{\omega}^{+}$and $\bar{\phi}$

$t_{\omega}^{d n} \quad$ Bilinear variable equal to product of $\Delta_{\omega}^{-}$and $\phi$

Set

$\Omega \quad$ Set of decision variables

Operators

I(condition) "If" operator

$\mathbb{E}$ (expression) "Expectation" operator.

\section{INTRODUCTION}

$\mathbf{I}$ $\mathrm{N}$ electricity markets, renewable generation has been increasing substantially following decreasing technology costs and supportive government policies [1]. In the European Union, the share of renewable generation has increased by 64\% from 2007 to 2017 [2]. Reference [3] reports $25 \mathrm{TWh}$ production from renewable generation in the third quarter of 2018 in UK (a 10\% increase year-on-year). A similarly increasing pattern is reported for the US electricity system [4]. This increasing share of renewable generation brings major environmental benefits but it also introduces new operational challenges. Although renewable generation has low marginal costs and is $\mathrm{CO}_{2}$ free, it is highly variable and intermittent [5]. This feature implies a need for more real-time balancing services by the system operator (SO). Authors in [6] and [7] discuss the balancing task of the SO in an electricity system with high penetration of intermittent-renewable generation. They suggest that at some point the intermittent-renewable generators will have to actively participate in the balancing market (BM) and be part of the solution as well as the cause. On the legislation side, the European Union directive 2003/54/EC enforces the non-discriminatory and marketbased provision of balancing services [8]. This encourages intermittent-renewable generators to provide balancing services in the BM (see for example [9] for Britain and [10] for Nordic countries).

Accordingly, authors in [11] propose different models for the SO to facilitate the active participation of wind generators in the balancing markets. In [12], a Lyapunov optimization based real-time strategy has been proposed in order to minimize the imbalance costs for a virtual power plant. This formulation does not need the specification of the probability distributions of the renewable power generation. In [13], a combined energy and reserve market model has been proposed to increase the wind producers' revenue by allowing them to optimally bid in both markets and help them reduce their imbalance costs. A mathematical model to maximize the overall profit of a portfolio of a wind farm and a combined heat and power by minimizing their imbalances in a two-price balancing market has been proposed in [14]. A bidding strategy to minimize the imbalance costs for wind power producers is described in [15] where forecast errors in wind power are represented as stochastic processes. But the risk associated with wind power forecast errors is not accounted for in this model. Reference [16] discusses the lack of economic incentives for the wind power producers in Portugal to minimize their imbalances as the wind imbalance costs are socialised. In [17], a method to choose the generation adjustments and the volumes of elastic demand that match the imbalances in the net inelastic demand (inelastic demand minus RES production), with respect to the day-ahead scheduling, has been proposed. In [18], the link between the power system cost due to wind power imbalances and the imbalance settlement payments to wind power producers is investigated. They draw a comparison between the one and two-price settlement systems.

TABLE I

OVERVIEW OF LITERATURE IN RELATED AREAS

\begin{tabular}{|c|c|c|c|c|c|c|c|}
\hline \multirow[t]{2}{*}{ Ref. } & \multirow{2}{*}{$\begin{array}{l}\text { BMC } \\
+ \text { ISC }\end{array}$} & \multicolumn{2}{|c|}{ Imbal. pricing } & \multirow{2}{*}{$\begin{array}{l}\text { Role of } \\
\text { SO } \\
\text { in BM }\end{array}$} & \multirow{2}{*}{$\begin{array}{l}\text { Prob. } \\
\text { constr. }\end{array}$} & \multirow{2}{*}{$\begin{array}{l}\text { Stoch. } \\
\text { gen. }\end{array}$} & \multirow{2}{*}{$\begin{array}{l}\text { Prob. } \\
\text { scen. } \\
\text { gen. }\end{array}$} \\
\hline & & Single & Dual & & & & \\
\hline$|\overline{6}|$ & $x$ & $x$ & $x$ & $x$ & $x$ & $x$ & $x$ \\
\hline [11] & $x$ & $\checkmark$ & $\checkmark$ & $x$ & $x$ & $x$ & $x$ \\
\hline 15 & $x$ & $\checkmark$ & $x$ & $x$ & $x$ & $\checkmark$ & $x$ \\
\hline 19 & $x$ & $\checkmark$ & $x$ & $x$ & $\checkmark$ & $\checkmark$ & $x$ \\
\hline 20 & $x$ & $x$ & $x$ & $\checkmark$ & $x$ & $x$ & $x$ \\
\hline |21] & $x$ & $x$ & $x$ & $\checkmark$ & $x$ & $x$ & $x$ \\
\hline [22] & $x$ & $x$ & $x$ & $\checkmark$ & $x$ & $x$ & $x$ \\
\hline [23] & $x$ & $\checkmark$ & $\checkmark$ & $x$ & $x$ & $\checkmark$ & $x$ \\
\hline 24 & $x$ & $\checkmark$ & $x$ & $x$ & $x$ & $x$ & $x$ \\
\hline 25 & $x$ & $x$ & $\checkmark$ & $x$ & $x$ & $x$ & $\checkmark$ \\
\hline 26 & $x$ & $\checkmark$ & $x$ & $x$ & $x$ & $x$ & $x$ \\
\hline Our & $\checkmark$ & $\checkmark$ & $\checkmark$ & $\checkmark$ & $\checkmark$ & $\checkmark$ & $\checkmark$ \\
\hline
\end{tabular}

Table II summarizes an overview of the published research on the various aspects of specifications. The first column in Table I. 'BMC+ISC' refers to the approach of considering both balancing market cost and imbalance settlement cost to take the balancing market decisions. The second column, 'Imbal. pricing (Single and Dual)', mentions the imbalance pricing schemes. While some of the literature is limited to the producers'/consumers' perspectives of minimizing the imbalance settlement costs, the third column, 'Role of SO in BM', aims to highlight which papers have discussed about the involvement of the SO in the balancing market. 'Prob. const.' (Probabilistic constraint) emphasizes the approach for considering the uncertainty in the system, which in our case is a CVaR (Conditional Value at Risk) constraint. The fifth column, 'Stoch. gen.' (Stochastic generation), highlights those papers that have considered stochastic generation in their work. 'Prob. scen. gen.' (Probabilistic scenario generation) tries to show if the method chosen for generating scenarios is probabilistic or not.

The above research generally, but implicitly, recognizes the role of the imbalance settlement cost (ISC) influencing the active participation of the intermittent-renewable generators in the BM. This point is further explained in $|6|$ and [20]. Accordingly, proper modeling of the ISC in the SO 
optimal-dispatch can ensure the efficient participation of the intermittent-renewable generators in the balancing market and maybe incentivize the intermittent-renewable generators to adapt technologies to reduce their ISC exposure (eg. hybrid facilities with battery-storage devices) or to hedge more effectively (eg. with weather derivatives). Furthermore, in principle, the $\mathrm{SO}$ as a regulated entity would be expected to perform a welfare maximizing approach to system balancing, and thus include all relevant costs to participants, including ISCs, but in practice this is not yet widespread. Hence this is the motivation for this paper.

\section{BACKGROUND}

In this section, we present a brief description of a few electricity markets to explain their basic functioning:

- Nordic electricity market: At the day-ahead (DA) stage, all the producers and consumers willing to participate in the DA market submit their bidding curves to the electricity market operator. Then the market operator clears the DA market based on uniform price auction and defines the DA price, all the producers and consumers below that price are cleared in the DA auction. Based on the updated forecasts, these producers and consumers can adjust their position in the market by trading in the intraday market. At the gate closure (60 minutes before actual delivery of electricity), the position becomes binding and cannot be changed further. However, at the time of actual delivery of electricity, these producers and consumers deviate from their scheduled value. It is then the responsibility of the SO to activate bids in the balancing market to match the supply and demand of electricity. According to the existing method, there is a merit-order dispatch (marginal cost pricing (MCP) auction) performed by the $\mathrm{SO}$, in the balancing market 1 to decide which producer or consumer needs to be called upon, based on their marginal cost of production or marginal utility of consumption. In the imbalance settlement stage, the SO calculates the imbalance settlement cost for each producer or consumer who participated in the market based on their deviation from the committed value and the direction of the system imbalance [29].

- Great Britain (GB): In GB electricity market, there is continuous trading up until the gate closure, which happens one hour before the actual delivery of electricity, and that is when all generators and retailers must nominate to the SO what they expect to generate or consume in the delivery period. But the participants are allowed to trade even after the gate closure for a further hour until the beginning of the actual delivery period. The delivery period length (also known as Settlement Period (SP)) is 30 minutes. It is the responsibility of the SO to ensure 50 $\mathrm{Hz}$ frequency, by activating bids and offers continuously

${ }^{1}$ Here we refer to the m-FRR (manual Frequency Restoration Reserve) of the balancing market. This is the main balancing resource in the Nordic market apart from this the other reserves are FCR-N (Frequency Containment Reserve-Normal), FCR-D (Frequency Containment Reserves-Disturbance) and a-FRR (automatic Frequency Restoration Reserve). For more information check [27], [28]. in every $\mathrm{SP}^{2}$. For each SP, a single imbalance price (SIP) is calculated. Based on whether the offer volumes exceed the bid volumes or vice versa, the NIV (Net Imbalance Volume) can be either positive or negative. The marginal price for $1 \mathrm{MW}$ of the NIV gives the SIP [19], [31].

- PJM 3. PJM compiles the bids in ascending price order and auction is performed to clear the bids until the point of intersection of the supply-demand curve. This sets the DA price for a particular hour for the delivery of electricity on the next day. Real-time market is another market that serves electricity for immediate delivery. Supply and demand curves are paired and based on the intersection point (MCP auction), the prices are calculated every five minutes. Based on the situation of fluctuations in the system, PJM informs the producers what their output should be. But if they deviate from PJM's instructions, they are penalized. If they follow the instructions of PJM, they are compensated for that. Another important market in PJM is the ancillary services market, which comprises of regulation and reserves market. The regulation market is in place to correct the small fluctuations in the electricity supply/demand that can affect the stability of power system. It allows to compensate those producers/consumers that have the ability to adjust their production/consumption as per the requirement in the system [33].

\section{CONTRIBUtions}

This paper contributes to the relevant research in this context in several ways:

- Our paper proposes a mechanism for the SO to dispatch generators to activate their up- and down-regulation bids on the basis of having lowest expected costs, taking into account both their production costs and potential deviations from nominated outputs. We propose a stochastic framework for clearing the real time balancing market by the SO.

- In our formulation, we introduce a CVaR constraint, which allows surplus generation or unmet demand, with an appropriate small probability. This produces useful and computationally tractable solutions under supply side uncertainty, where hard constraints with no surplus generation or unmet demand might lead to infeasibility.

- The proposed method has been mathematically applied to compare three imbalance pricing models namely Dual Imbalance Pricing (DIP), Single Imbalance Pricing with Spot Reversion (SIP-SR) and Single Imbalance Pricing (SIP) models.

- The three proposed models have been tested on a stylised five-generator system and a more realistic eight-generator

\footnotetext{
${ }^{2}$ Short term operating reserve (STOR) provides the grid in GB with additional power when actual demand in the network is greater than the forecast and/or there is unforeseen generation unavailability [30].

${ }^{3} \mathrm{PJM}$ : Pennsylvania-Jersey-Maryland, which is the regional transmission organiziation that is responsible for coordinating the wholesale electricity market in some or all parts of Indiana, Delaware, Virginia, West Virginia, Maryland, Illinois, Pennsylvania, Michigan, Kentucky, North Carolina, Tennessee, New Jersey, Ohio, and the District of Columbia [32].
} 


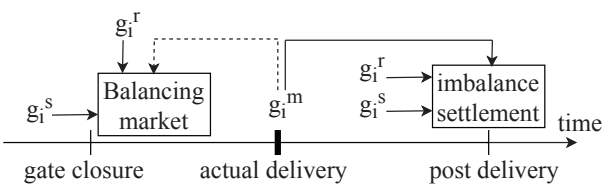

Fig. 1. The proposed balancing market operation (Dotted line represents uncertainty)

system. In the case of eight-generator system, the uncertain parameters in the stochastic MILP models were estimated and predicted using a state-of-the art algorithm, the Generalized Additive Models for Location, Scale and Shape (GAMLSS). We demonstrate the performance of our proposed MILP models and the GAMLSS-based uncertainty-modeling algorithm with realistic numerical studies.

The rest of the paper is structured as follows. Section IV develops the optimal-dispatch model for the SO. Section V presents the GAMLSS-based forecasting model for generation imbalances. An illustrative example is provided in Section VI. Numerical results based on variations of a Nordic case study is provided in Section VII Finally, Section VIII concludes the paper.

\section{MATHEMATiCAl Formulation}

We assume three stages in our analysis. At gate closure the scheduled generations $\left(g_{i}^{s}\right)$ are reported to the SO. Then the $\mathrm{SO}$ receives up-regulation offers from generators for increasing output and down-regulation bids for reducing output, $\left(g_{i}^{r}\right)$. If called, generators receive payments for their generation, or pay for their reduced generation. The SO also forecasts system demand in real time and estimates the imbalance settlement charges. Since the metered output of generators $\left(g_{i}^{m}\right)$ are available only at the imbalance settlement stage, they are uncertain parameters in balancing-market optimal dispatch model. These stages are illustrated in Fig. 1. We now consider three variations in the formation of imbalance charges.

\section{A. Dual Imbalance Pricing (DIP)}

The DIP mechanism uses two imbalance prices, often called system buy price (SBP) and system sell price (SSP) [34]. The SBP is the average price at which the system has to buy electricity during a balancing period and the SSP reflects the average price at which the system has to sell electricity in order to dispose of surplus energy. Participants will then pay SBP or receive SSP depending upon whether their imbalance volumes were short or long. This mechanism has been favoured by regulators who want to deter speculation. It was the mechanism introduced in Great Britain in 2001 (although it has since been changed), also in France, Spain, Italy and elsewhere. At gate closure, let $\lambda_{i}^{u p}, \lambda_{i}^{d n}, g_{i}^{u p}$ and $g_{i}^{d n}$ represent bid prices and volumes for generator $i$ participating in the balancing market. For notational simplicity, we assume that each generator submits only one bid pair. Let $\phi_{i}^{u p}$ (respectively, $\phi_{i}^{d n}$ ) be binary variables which take values 1 or 0 depending whether the bid (respectively, offer) from generator $i$ is accepted or not.

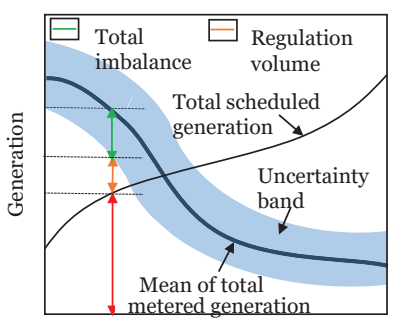

Time

(a)

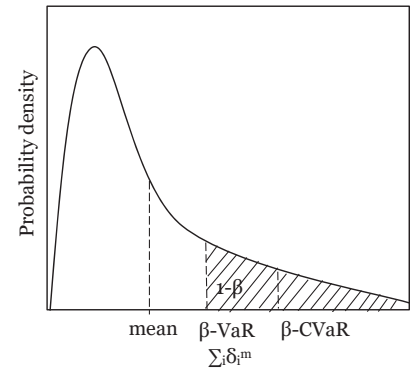

(b)
Fig. 2. (a) Time series for scheduled generation and depiction of imbalance at a specific time (b) Illustration of $\delta_{i}^{m}$

1) Objective function of proposed optimal-dispatch model: The proposed objective function for the SO optimal-dispatch model is given by (1), where BSC stands for Balance Settlement Cost:

$$
\begin{aligned}
& \mathrm{BSC}=\sum_{i}\left(\lambda_{i}^{u p} g_{i}^{u p} \phi_{i}^{u p}-\lambda_{i}^{d n} g_{i}^{d n} \phi_{i}^{d n}\right)+ \\
& \max \left(\sum_{i} \delta_{i}^{m}, 0\right) \frac{\sum_{i} \lambda_{i}^{d n} g_{i}^{d n} \phi_{i}^{d n}}{\sum_{i} g_{i}^{d n} \phi_{i}^{d n}} \mathbb{I}\left(\sum_{i} \phi_{i}^{d n} \geq 1\right)+ \\
& \min \left(\sum_{i} \delta_{i}^{m}, 0\right) \frac{\sum_{i} \lambda_{i}^{u p} g_{i}^{u p} \phi_{i}^{u p}}{\sum_{i} g_{i}^{u p} \phi_{i}^{u p}} \mathbb{I}\left(\sum_{i} \phi_{i}^{u p} \geq 1\right)
\end{aligned}
$$

In (1), both $g_{i}^{u p}$ and $g_{i}^{d n}$ are assumed to be positive. $g_{i}^{m}$ is the metered output of generator $i$ and is considered to be a random parameter with known means and known continuous joint distribution. $g_{i}^{s}$ is the scheduled power dispatch of generator $i$. The $\delta_{i}^{m}=g_{i}^{m}-\left(g_{i}^{s}+g_{i}^{u p} \phi_{i}^{u p}-g_{i}^{d n} \phi_{i}^{d n}\right)$ models the deviation for generator $i$. The SO objective function in (1) can be split into two parts, $1 \mathrm{a}$ is the Balancing Market Cost (BMC) while (1b) and (1c) represent the positive and negative Imbalance Settlement Cost (ISC), respectively. Observe that whilst ISCs are the charges incurred by the generators, they are also the recourse costs incurred by the SO in balancing, because of the non-firm regulating dispatch.

2) Constraints of proposed optimal-dispatch model: Let $f\left(\phi, g^{m}\right)=\sum_{i} \delta_{i}^{m}=\sum_{i}\left(g_{i}^{m}-\left(g_{i}^{s}+g_{i}^{u p} \phi_{i}^{u p}-g_{i}^{d n} \phi_{i}^{d n}\right)\right)$ be the deviation function, where $\phi$ and $g^{m}$ are vector valued variables corresponding to $\phi_{i}$ and $g_{i}^{m}$. Fig. $2 \mathrm{a}$ depicts the time series for total scheduled generation and the mean of the total metered generation. The blue color band highlights the uncertainty in the total metered generation. At a given time instant, the red colored line represents the total scheduled generation at that time, orange colored line gives the regulation volume, and green colored line shows the total imbalance (deviation) at that time. Our paper discusses one such specific time instant. In Fig. 2b, the probability density of the total imbalance (deviation) is demonstrated, the details about which are discussed further in this section. In our formulation, we allow surplus generation $\left(f\left(\phi, g^{m}\right)>0\right)$ or unmet demand $\left(f\left(\phi, g^{m}\right)<0\right)$ with an appropriate small probability. The probability of $f\left(\phi, g^{m}\right)$ not exceeding a given $\alpha$ is given by:

$$
\bar{\Psi}(\phi, \alpha)=\int_{f\left(\phi, g^{m}\right) \leq \alpha} p\left(g^{m}\right) d g^{m}
$$


where $p\left(g^{m}\right)$ is the joint distribution function of $g^{m}$. Similarly, the probability of $-f$ not exceeding a given $\alpha$ ( $f$ exceeding $-\alpha)$ is given by:

$$
\underline{\Psi}(\phi, \alpha)=\int_{-f\left(\phi, g^{m}\right) \leq \alpha} p\left(g^{m}\right) d g^{m}
$$

For a specified probability level $\beta$, let the surplus generation be defined by:

$$
\bar{\alpha}_{\beta}(\phi):=\min \left\{\alpha \in \mathbf{R}_{+}, \bar{\Psi}(\phi, \alpha) \geq \beta\right\}
$$

and the unmet level of demand is defined by:

$$
\underline{\alpha}_{\beta}(\phi):=\min \left\{\alpha \in \mathbf{R}_{+}, \underline{\Psi}(\phi, \alpha) \geq \beta\right\}
$$

Thus, given a probability level $\beta$, the surplus generation does not exceed $\bar{\alpha}$ with probability $\beta$. In other words, $\bar{\alpha}$ is one endpoint of the non-empty interval of values $\alpha$ such that $\bar{\Psi}(\phi, \alpha)=\beta$. Similarly, unmet demand does not exceed $\underline{\alpha}$ with probability $\beta$. We then define the average surplus generation if it already exceeds $\bar{\alpha}$ as:

$$
\overline{\mathcal{L}}_{\beta}(\phi)=(1-\beta)^{-1} \int_{f\left(\phi, g^{m}\right) \geq \bar{\alpha}_{\beta}(\phi)} f\left(\phi, g^{m}\right) p\left(g^{m}\right) d g^{m}
$$

Similarly, we define the average unmet demand if it already exceeds $\underline{\alpha}$ as:

$$
\underline{\mathcal{L}}_{\beta}(\phi)=(1-\beta)^{-1} \int_{-f\left(\phi, g^{m}\right) \geq \underline{\alpha}_{\beta}(\phi)} f\left(\phi, g^{m}\right) p\left(g^{m}\right) d g^{m}
$$

Note that the probability of $f\left(\phi, g^{m}\right)$ exceeding $\bar{\alpha}$ is $1-\beta$, so that $\bar{L}_{\beta}(\phi)$ gives conditional expectation, i.e., the average surplus generation, when it exceeds $\bar{\alpha}$. Similar comments hold for $\underline{L}_{\beta}(\phi)$.

$\bar{L}_{\beta}(\phi)$ and $\underline{L}_{\beta}(\phi)$ can be incorporated into our optimization model through appropriately relaxed versions of these functions. These are defined as follows:

$$
\begin{aligned}
& \bar{F}_{\beta}(\phi, \bar{\alpha})=\bar{\alpha}+(1-\beta)^{-1} \int_{g^{m}}\left(f\left(\phi, g^{m}\right)-\bar{\alpha}\right)_{+} d g^{m} \\
& \underline{F}_{\beta}(\phi, \underline{\alpha})=\underline{\alpha}+(1-\beta)^{-1} \int_{g^{m}}\left(-f\left(\phi, g^{m}\right)-\underline{\alpha}\right)_{+} d g^{m}
\end{aligned}
$$

where $(x)_{+}=\max (x, 0)$. Then $\bar{F}_{\beta}(\phi, \bar{\alpha})$ (respectively $\left.\underline{F}_{\beta}(\phi, \underline{\alpha})\right)$ is convex and continuously differentiable in $\bar{\alpha}$ (respectively $\underline{\alpha}$ ). Further, $\overline{\mathcal{L}}_{\beta}(\phi)=\min _{\bar{\alpha} \in \mathbf{R}} \bar{F}_{\beta}(\phi, \bar{\alpha})$ and a similar result holds for $\underline{\mathcal{L}}_{\beta}(\phi)$ [35]. Now, if we generate samples of $g^{m}$, we can write an approximation to $\bar{F}_{\beta}(\phi, \bar{\alpha})$ as:

$$
\overline{\mathcal{F}}_{\beta}(\phi, \bar{\alpha})=\bar{\alpha}+\frac{1}{N(1-\beta)} \sum_{j}\left(f\left(\phi, \operatorname{vec}\left(g_{i, \omega}^{m}\right)\right)-\bar{\alpha}\right)_{+}
$$

where $\operatorname{vec}\left(x_{i}\right)$ represents a vector with $x_{i}$ as its $i^{\text {th }}$ element, with its dimension determined by the context. $\underline{\mathcal{F}}_{\beta}(\phi, \underline{\alpha})$ is defined similarly. For a given System Imbalance Level (SIL), $\overline{\mathcal{F}}_{\beta}(\phi, \bar{\alpha}) \leq S I L$ and $\underline{\mathcal{F}}_{\beta}(\phi, \underline{\alpha}) \leq S I L$ can be written as in (10).

$$
\begin{aligned}
& \bar{\alpha}+\frac{1}{N(1-\beta)}\left(f\left(\phi, \operatorname{vec}\left(g_{i, \omega}^{m}\right)\right)-\bar{\alpha}\right)_{+} \leq S I L \\
& \underline{\alpha}+\frac{1}{N(1-\beta)}\left(-f\left(\phi, \operatorname{vec}\left(g_{i, \omega}^{m}\right)\right)-\underline{\alpha}\right)_{+} \leq S I L
\end{aligned}
$$

where in $(10), S I L$ in $\mathrm{MWh}$ is a behavioural parameter to reflect SO aversion to large real-time imbalances. It represents the total imbalance in the system tolerated by the SO and risk constraints (2) represent the probabilistic tolerance of this large imbalance.

3) The proposed optimal-dispatch model: As we consider the measured generation $g_{i, \omega}^{m}$ to be an uncertain parameter, it can be modeled using a set of scenarios. We assume that scenarios of $g_{i, \omega}(\omega=1,2, \cdots, \mathcal{N})$ are available, along with the associated probability weights $\pi_{\omega}$. The discussion of how to generate scenarios is explained in Section V. Explicit modeling of the ISC in the optimization model (12) exposes the intermittentrenewable generators participating in the balancing market to their imbalance penalties (because of the deviation from their nominated outputs). The deviation $\delta_{i, \omega}^{m}$ depends on the stochastic measured generation $g_{i, \omega}^{m}$ and the expression of deviation can be given as: $\delta_{i, \omega}^{m}=g_{i, \omega}^{m}-\left(g_{i}^{s}+g_{i}^{u p} \phi_{i}^{u p}-g_{i}^{d n} \phi_{i}^{d n}\right)$. This term of $\delta_{i, \omega}^{m}$ when introduced in $[1 \mathrm{~b}$ ) and $\sqrt{1 \mathrm{c}}$ motivates us to take the expected value of the expression in (1b) and (1c) due to the stochasticity. Therefore, the expression of BSC becomes (11):

$$
\begin{aligned}
& \mathrm{BSC}=\sum_{i}\left(\lambda_{i}^{u p} g_{i}^{u p} \phi_{i}^{u p}-\lambda_{i}^{d n} g_{i}^{d n} \phi_{i}^{d n}\right)+ \\
& \mathbb{E}\left(\max \left(\sum_{i} \delta_{i, \omega}^{m}, 0\right) \frac{\sum_{i} \lambda_{i}^{d n} g_{i}^{d n} \phi_{i}^{d n}}{\sum_{i} g_{i}^{d n} \phi_{i}^{d n}} \mathbb{I}\left(\sum_{i} \phi_{i}^{d n} \geq 1\right)\right)+ \\
& \mathbb{E}\left(\min \left(\sum_{i} \delta_{i, \omega}^{m}, 0\right) \frac{\sum_{i} \lambda_{i}^{u p} g_{i}^{u p} \phi_{i}^{u p}}{\sum_{i} g_{i}^{u p} \phi_{i}^{u p}} \mathbb{I}\left(\sum_{i} \phi_{i}^{u p} \geq 1\right)\right)
\end{aligned}
$$

Here it can be observed that $(11 \mathrm{~b})$ and $(11 \mathrm{c})$ together comprise the expected value of ISC.

Hence, the proposed optimization model for SO considering both BMC and ISC can be presented as in 12 .

$$
\begin{aligned}
& \underset{\Omega_{1}=\left\{\phi_{i}^{u p}, \phi_{i}^{d n}, \underline{\alpha}, \bar{\alpha}\right\}}{\operatorname{Minimize}} \mathrm{BSC}=\mathrm{BMC}+\mathbb{E}(\mathrm{ISC}) \\
& \text { subject to: } 10,,\left\{\phi_{i}^{u p}, \phi_{i}^{d n}\right\} \in\{0,1\}, \underline{\alpha}, \bar{\alpha} \in \mathbf{R}, \forall i
\end{aligned}
$$

4) The MILP formulation of the proposed optimal-dispatch model: We now present a MILP model of the deterministic equivalent problem for (12). The total deviation in a scenario $w$ can be given as $\Delta_{\omega}=\sum_{i} \delta_{i, \omega}^{m}$ where $\delta_{i, \omega}^{m}=g_{i, \omega}^{m}-\left(g_{i}^{s}+\right.$ $\left.g_{i}^{u p} \phi_{i}^{u p}-g_{i}^{d n} \phi_{i}^{d n}\right)$. The $\Delta_{\omega}^{+}=\operatorname{Max}\left(\Delta_{\omega}, 0\right)$ can be linearized as shown in (13).

$$
\begin{aligned}
& 0 \leq \Delta_{\omega}^{+} \leq b_{\omega}^{+} \Xi^{(1)} \\
& \Delta_{\omega} \leq \Delta_{\omega}^{+} \leq \Delta_{\omega}+\left(1-b_{\omega}^{+}\right) \Xi^{(1)}
\end{aligned}
$$

where $\Xi^{(1)}$ is a suitable large constant. The $\Delta_{\omega}^{-}=\operatorname{Max}\left(-\Delta_{\omega}, 0\right)$ can be linearized similarly as in (13). Referring to the positive deviation part of 11 , we denote $c_{\omega}=\frac{\sum_{i} \lambda_{i}^{d n} g_{i}^{d n} \phi_{i}^{d n} \Delta_{\omega}^{+}}{\sum_{i} g_{i}^{d n} \phi_{i}^{d n}}$ which can be written as (14):

$$
c_{\omega} \sum_{i} g_{i}^{d n} \phi_{i}^{d n}=\sum_{i} \lambda_{i}^{d n} g_{i}^{d n} \phi_{i}^{d n} \Delta_{\omega}^{+}
$$

Using change of variable $I_{i, w}^{d n}=c_{\omega} \phi_{i}^{d n}$ in $\sqrt{14}$, the non-linear term can be linearized by implementing [15]:

$$
\begin{aligned}
& 0 \leq I_{i, w}^{d n} \leq \phi_{i}^{d n} \Xi^{(2)} \\
& c_{\omega}-\left(1-\phi_{i}^{d n}\right) \Xi^{(2)} \leq I_{i, w}^{d n} \leq c_{\omega}
\end{aligned}
$$


where $\Xi^{(2)}$ is a suitably large constant. Also, substituting $I_{i, w}^{+}=\phi_{i}^{d n} \Delta_{\omega}^{+}$in 14 , it can be linearized as in 15 . The linearized form of (14), after implementing the change of variable, can be written as in 16 :

$$
\sum_{i} g_{i}^{d n} I_{i, w}^{d n}=\sum_{i} \lambda_{i}^{d n} g_{i}^{d n} I_{i, w}^{+}
$$

Consider the negative deviation part of (1), we define $s_{\omega}=$ $\frac{\sum_{i} \lambda_{i}^{u p} g_{i}^{u p} \phi_{i}^{u p} \Delta_{\omega}^{-}}{\sum_{i} g_{i}^{u p} \phi_{i}^{u p}}$ which means:

$$
s_{\omega} \sum_{i} g_{i}^{u p} \phi_{i}^{u p}=\sum_{i} \lambda_{i}^{u p} g_{i}^{u p} \phi_{i}^{u p} \Delta_{\omega}^{-}
$$

By implementing the substitutions, $I_{i, w}^{u p}=s_{\omega} \phi_{i}^{u p}$ and $I_{i, w}^{-}=\phi_{i}^{u p} \Delta_{\omega}^{-}$, the linearized form of 177 can be written as $\sum_{i} g_{i}^{u p} I_{i, w}^{u p}=\sum_{i} \lambda_{i}^{u p} g_{i}^{u p} I_{i, w}^{-}$by following the similar equations as in (15). The If condition in (1b) is written as linear constraints $\sum_{i} \phi_{i}^{d n} \geq 1-\Xi^{(3)}(1-\underline{\phi})$ and $\sum_{i} \phi_{i}^{d n} \leq$ $1+\Xi^{(3)}(\underline{\phi})$. A similar linearization can be adopted for $1 \mathrm{c}$ and replaced with $\bar{\phi}$. Then the objective function becomes:

$$
\begin{aligned}
& \underset{\Omega_{2}}{\operatorname{Minimize}} \sum_{i}\left(\lambda_{i}^{u p} g_{i}^{u p} \phi_{i}^{u p}-\lambda_{i}^{d n} g_{i}^{d n} \phi_{i}^{d n}\right)+ \\
& \sum_{\omega} \pi_{\omega}\left(\underline{\phi} c_{\omega}-\bar{\phi} s_{\omega}\right)
\end{aligned}
$$

where $\Omega_{2}=\Omega_{1} \cup\left\{\bar{\phi}, \underline{\phi}, b_{\omega}^{+}, b_{\omega}^{-}, c_{\omega}, s_{\omega}, \Delta_{\omega}^{+}, \Delta_{\omega}^{-}, I_{i, w}^{d n}, I_{i, w}^{u p}\right.$, $\left.I_{i, w}^{-}, I_{i, w}^{+}\right\}$. If we use $z_{\omega}^{d n}=\phi c_{\omega}$ and $z_{\omega}^{u p}=\bar{\phi} s_{\omega}$, then the nonlinear expressions $\left(\phi c_{\omega}\right.$ and $\left.\bar{\phi} s_{\omega}\right)$ can be linearized as in (15). The probabilistic constraint can be written as:

$$
\bar{\alpha}+\frac{1}{(1-\beta)} \sum_{\omega}^{N} \pi_{\omega} u_{\omega}^{+} \leq S I L
$$

where $u_{\omega}^{+}=\operatorname{Max}\left(\Delta_{\omega}^{+}-\bar{\alpha}, 0\right)$. Also,

$$
\underline{\alpha}+\frac{1}{(1-\beta)} \sum_{\omega}^{N} \pi_{\omega} u_{\omega}^{-} \leq S I L
$$

where $u_{\omega}^{-}=\operatorname{Max}\left(\Delta_{\omega}^{-}-\underline{\alpha}, 0\right)$.

Linearization of $\operatorname{Max}(\cdot)$ operator is possible in similar way as in (13). Accordingly, the optimization problem (12) can be re-formulated as the stochastic mixed-integer linear program (MILP) in 21):

$\underset{\Omega_{A}}{\operatorname{Minimize}} \sum_{i}\left(\lambda_{i}^{u p} g_{i}^{u p} \phi_{i}^{u p}-\lambda_{i}^{d n} g_{i}^{d n} \phi_{i}^{d n}\right)+\sum_{\omega} \pi_{\omega}\left(z_{\omega}^{d n}-z_{\omega}^{u p}\right)$

subject to : 13), 15, $, 16,, 19,, 20$,

$\left\{\bar{\phi}, \underline{\phi}, \phi_{i}^{u p}, \phi_{i}^{d n}, b_{\omega}^{+}, b_{\omega}^{-}\right\} \in\{0,1\} \forall i, \omega$

where $\Omega_{A}=\Omega_{2} \cup\left\{z_{\omega}^{u p}, z_{\omega}^{d n}, u_{\omega}^{+}, u_{\omega}^{-}\right\}$is the set of decision variables in 21].

\section{B. Benchmark model for the DIP model}

An illustration of the benchmark model for the DIP model is given in Fig. 3 In the benchmark model, the decision of the SO to activate up- or down-regulation bids is taken solely based on the marginal cost of the generators. This is achieved by minimizing expression 11a. The output of the optimization problem would then give the values for $\phi_{i}^{u p}$ and $\phi_{i}^{d n}$. These

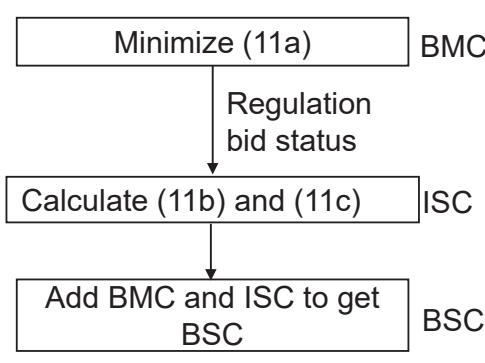

Fig. 3. Illustration of Benchmark model for the DIP model

values serve as inputs to the calculation of $11 \mathrm{~b})$ and $(11 \mathrm{c})$, which collectively give the value for ISC. The optimized value for BMC and the calculated value of ISC can be added together to find the BSC.

\section{Single Imbalance Pricing with Spot Reversion (SIP-SR) Model}

The SIP-SR mechanism differs in two respects from above. It is based on marginal rather than average pricing, i.e. the imbalance price is set according to the most expensive generator that is cleared during up-regulation hour, or the cheapest generator cleared during down-regulation hour. Further, as shown in Table III if the deviations contribute to the system imbalance then the balance provider is charged according to the regulation price. However, if the deviations help to maintain the system in balance then it should not be penalized and accordingly the spot market price is applied [29]. This is the current Nordic model.

TABLE II

PRICES IN BALANCING MARKET FOR SIP-SR MODEL

\begin{tabular}{lll}
\hline \hline & Up-regulation hour & Down-regulation hour \\
\hline$\Delta_{\omega}^{-}$ & $\mathrm{p}=\lambda_{\text {reg }}^{\text {up }}$ & $\mathrm{p}=\lambda^{\text {spot }}$ \\
$\Delta_{\omega}^{+}$ & $\mathrm{p}=\lambda^{\text {spot }}$ & $\mathrm{p}=\lambda_{\text {reg }}^{\text {dn }}$ \\
\hline
\end{tabular}

The objective function of SIP-SR optimal dispatch model can be written as:

$$
\begin{aligned}
& \underset{\Omega_{3}}{\operatorname{Minimize}} \sum_{i}\left(\lambda_{i}^{u p} g_{i}^{u p} \phi_{i}^{u p}-\lambda_{i}^{d n} g_{i}^{d n} \phi_{i}^{d n}\right)+ \\
& \sum_{\omega} \pi_{\omega}\left[\Delta_{\omega}^{+} \lambda^{s p o t} \mathbb{I}\left(\sum_{i} \phi_{i}^{u p} g_{i}^{u p}>\sum_{i} \phi_{i}^{d n} g_{i}^{d n}\right)+\right. \\
& \Delta_{\omega}^{+} \lambda^{s p o t} \mathbb{I}\left(\sum_{i} \phi_{i}^{u p} g_{i}^{u p}=\sum_{i} \phi_{i}^{d n} g_{i}^{d n}\right)+ \\
& \Delta_{\omega}^{+} \lambda_{r e g}^{d n} \mathbb{I}\left(\sum_{i} \phi_{i}^{u p} g_{i}^{u p}<\sum_{i} \phi_{i}^{d n} g_{i}^{d n}\right)- \\
& \Delta_{\omega}^{-} \lambda_{r e g}^{u p} \mathbb{I}\left(\sum_{i} \phi_{i}^{u p} g_{i}^{u p}>\sum_{i} \phi_{i}^{d n} g_{i}^{d n}\right)- \\
& \Delta_{\omega}^{-} \lambda^{s p o t} \mathbb{I}\left(\sum_{i} \phi_{i}^{u p} g_{i}^{u p}<\sum_{i} \phi_{i}^{d n} g_{i}^{d n}\right)- \\
& \left.\Delta_{\omega}^{-} \lambda^{s p o t} \mathbb{I}\left(\sum_{i} \phi_{i}^{u p} g_{i}^{u p}=\sum_{i} \phi_{i}^{d n} g_{i}^{d n}\right)\right]
\end{aligned}
$$

$\Omega_{3}=\left\{\phi_{i}^{u p}, \phi_{i}^{d n}, \lambda_{\text {reg }}^{d n}, \lambda_{\text {reg }}^{u p}, \Delta_{\omega}^{+}, \Delta_{\omega}^{-}\right\}$where $\lambda_{\text {reg }}^{d n}=$ $\operatorname{Min}\left(\lambda_{i}^{d n} \phi_{i}^{d n}\right)$ and $\lambda_{\text {reg }}^{u p}=\operatorname{Max}\left(\lambda_{i}^{u p} \phi_{i}^{u p}\right)$. Hence, the objective function (22) becomes: 


$$
\begin{aligned}
& \underset{\Omega_{3}}{\operatorname{Minimize}}\left(\sum_{i}\left(\lambda_{i}^{u p} g_{i}^{u p} \phi_{i}^{u p}-\lambda_{i}^{d n} g_{i}^{d n} \phi_{i}^{d n}\right)+\right. \\
& \sum_{\omega} \pi_{\omega}\left[\Delta_{\omega}^{+} \lambda^{s p o t} \mathbb{I}\left(\sum_{i} \phi_{i}^{u p} g_{i}^{u p}>\sum_{i} \phi_{i}^{d n} g_{i}^{d n}\right)+\right. \\
& \Delta_{\omega}^{+} \lambda^{s p o t} \mathbb{I}\left(\sum_{i} \phi_{i}^{u p} g_{i}^{u p}=\sum_{i} \phi_{i}^{d n} g_{i}^{d n}\right)+ \\
& \operatorname{Min}\left(\lambda_{i}^{d n} \phi_{i}^{d n} \Delta_{\omega}^{+}\right)-\operatorname{Max}\left(\lambda_{i}^{u p} \phi_{i}^{u p} \Delta_{\omega}^{-}\right) \\
& -\Delta_{\omega}^{-} \lambda^{s p o t} \mathbb{I}\left(\sum_{i} \phi_{i}^{u p} g_{i}^{u p}<\sum_{i} \phi_{i}^{d n} g_{i}^{d n}\right)- \\
& \left.\left.\Delta_{\omega}^{-} \lambda^{s p o t} \mathbb{I}\left(\sum_{i} \phi_{i}^{u p} g_{i}^{u p}=\sum_{i} \phi_{i}^{d n} g_{i}^{d n}\right)\right]\right)
\end{aligned}
$$

If $N_{i, \omega}^{d n}=\phi_{i}^{d n} \Delta_{\omega}^{+}$and $N_{i, \omega}^{u p}=\phi_{i}^{u p} \Delta_{\omega}^{-}$, the non-linearity introduced (bilinear term $\phi_{i}^{d n} \Delta_{\omega}^{+}$and $\phi_{i}^{u p} \Delta_{\omega}^{-}$) can be linearized in the same way as 15 . The expression $c_{\omega}^{d n}=\operatorname{Min}\left(\lambda_{i}^{d n} N_{i, \omega}^{d n}\right)$ is linearized in 24) (the term $c_{\omega}^{u p}=\operatorname{Max}\left(\lambda_{i}^{u p} N_{i, \omega}^{u p}\right)$ can be linearized in the same way as shown in the Appendix):

$$
\begin{aligned}
& -q_{i \omega} \Xi^{(5)} \leq c_{\omega}^{d n}-\lambda_{i}^{d n} N_{i, \omega}^{d n} \leq q_{i \omega} \Xi^{(5)} \\
& \sum_{i} q_{i \omega}=\mathcal{G}-1
\end{aligned}
$$

where $\mathcal{G}$ is the total number of generators in the electricity market and $q_{i \omega}$ is a binary variable. The If condition, $\mathbb{I}\left(\sum_{i} \phi_{i}^{u p} g_{i}^{u p}>\sum_{i} \phi_{i}^{d n} g_{i}^{d n}\right)$ in 23 is replaced by binary variable $\bar{\phi}$ where:

$$
\begin{aligned}
\sum_{i} \phi_{i}^{u p} g_{i}^{u p} & \geq \sum_{i} \phi_{i}^{d n} g_{i}^{d n}-\Xi^{(6)}(1-\bar{\phi})+\epsilon \\
\sum_{i} \phi_{i}^{u p} g_{i}^{u p} & \leq \sum_{i} \phi_{i}^{d n} g_{i}^{d n}+\Xi^{(6)} \bar{\phi}-\epsilon
\end{aligned}
$$

where $\epsilon$ is a non-zero number. The If condition, $\mathbb{I}\left(\sum_{i} \phi_{i}^{u p} g_{i}^{u p}<\sum_{i} \phi_{i}^{d n} g_{i}^{d n}\right)$ in 23] can be linearized the same way as in (38) using binary variable $\phi$. The $I f$ condition, $\mathbb{I}\left(\sum_{i} \phi_{i}^{u p} g_{i}^{u p}=\sum_{i} \phi_{i}^{d n} g_{i}^{d n}\right)$ in 23 is linearized by replacing it by binary variable $\phi$ and rewriting the If condition as $\sum_{i} k_{i}^{u p} g_{i}^{u p}=\sum_{i} k_{i}^{d n} g_{i}^{d n}$ where $k_{i}^{u p}\left(=\phi \phi_{i}^{u p}\right)$ is linearized using $\phi+\phi_{i}^{u p}-1 \leq k_{i}^{u p} \leq \phi$ and $k_{i}^{u p} \leq \phi_{i}^{u p}$. The $k_{i}^{d n}=\phi \phi_{i}^{d n}$ can be linearized in the same way as $k_{i}^{u p}$. The objective function can now be written as:

$$
\begin{array}{r}
\underset{\Omega_{4}}{\operatorname{Minimize}} \sum_{i}\left(\lambda_{i}^{u p} g_{i}^{u p} \phi_{i}^{u p}-\lambda_{i}^{d n} g_{i}^{d n} \phi_{i}^{d n}\right)+\sum_{\omega} \pi_{\omega}\left[\Delta_{\omega}^{+} \lambda^{s p o t} \bar{\phi}\right. \\
\left.+\Delta_{\omega}^{+} \lambda^{s p o t} \phi+c_{\omega}^{d n}-c_{\omega}^{u p}-\Delta_{\omega}^{-} \lambda^{s p o t} \underline{\phi}-\Delta_{\omega}^{-} \lambda^{s p o t} \phi\right] .
\end{array}
$$

$\Omega_{4}=\Omega_{3} \cup\left\{\phi, \bar{\phi}, \underline{\phi}, b_{\omega}^{+}, b_{\omega}^{-}, N_{i, \omega}^{d n}, N_{i, \omega}^{u p}, c_{\omega}^{d n}, c_{\omega}^{u p}, k_{i}^{d n}, k_{i}^{u p}\right\}$ If we use $t_{\omega}^{u p}=\Delta_{\omega}^{+} \bar{\phi}, h^{+}=\Delta_{\omega}^{+} \phi, t_{\omega}^{d n}=\Delta_{\omega}^{-} \phi$, and $h^{-}=\Delta_{\omega}^{-} \phi$ then the non-linear expressions can be linearized similar to (15). Accordingly, the final stochastic MILP model is given in (27).

$$
\begin{aligned}
& \underset{\Omega_{B}}{\operatorname{Minimize}} \sum_{i}\left(\lambda_{i}^{u p} g_{i}^{u p} \phi_{i}^{u p}-\lambda_{i}^{d n} g_{i}^{d n} \phi_{i}^{d n}\right)+ \\
& \sum_{\omega} \pi_{\omega}\left[\lambda^{s p o t}\left(t_{\omega}^{u p}+h^{+}\right)+c_{\omega}^{d n}-c_{\omega}^{u p}-\lambda^{s p o t}\left(t_{\omega}^{d n}+h^{-}\right)\right] \\
& \text {subject to : } 13,,(19), 20],(24) \\
& \left\{\phi, \bar{\phi}, \underline{\phi}, \phi_{i}^{u p}, \phi_{i}^{d n}, b_{\omega}^{+}, b_{\omega}^{-}\right\} \in\{0,1\} \forall i, \omega
\end{aligned}
$$

Where $\Omega_{B}=\left\{\Omega_{4} \cup t_{\omega}^{u p}, t_{\omega}^{d n}, h^{+}, h^{-}, u_{\omega}^{+}, \bar{\alpha}, u_{\omega}^{-}, \underline{\alpha}\right\}$ is the set of decision variables in 27).

\section{Benchmark model for the SIP-SR model}

As explained in Subsection IV-B, the benchmark model for the SIP-SR model works on the similar principle. Fig. 4 illustrates the functioning of the benchmark model. The SO ignores the deviations of the generators while choosing them for up- or down-regulation. The decision of $\phi_{i}^{u p}$ and $\phi_{i}^{d n}$ is taken by minimizing (22a), which gives the BMC. Based on the output of the optimization problem, $22 \mathrm{~b},(22 \mathrm{c}),(22 \mathrm{~d}),(22 \mathrm{e}), 22 \mathrm{f})$, and $22 \mathrm{~g}$ ) are calculated to find the ISC. Thus, BMC and ISC are added together to get the BSC value for the benchmark model.

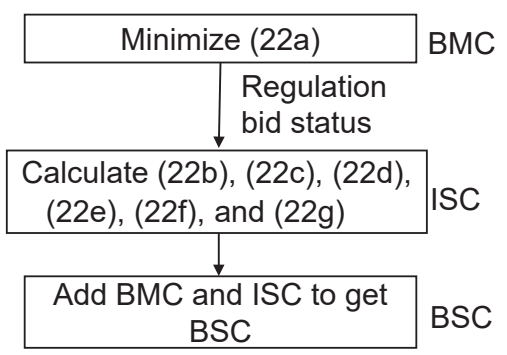

Fig. 4. Illustration of Benchmark model for the SIP-SR model

\section{E. Single Imbalance Pricing (SIP) Model}

The SIP model here, based on the imbalance pricing mechanism introduced in Great Britain (GB) in 2015, is summarized in Table III

TABLE III

PRICES IN BALANCING MARKET FOR SIP MODEL

\begin{tabular}{lll}
\hline \hline & Up-regulation hour & Down-regulation hour \\
\hline$\Delta_{\omega}^{-}$ & $\mathrm{p}=\lambda_{\text {reg }}^{u p}$ & $\mathrm{p}=\lambda_{r e g}^{d n}$ \\
$\Delta_{\omega}^{+}$ & $\mathrm{p}=\lambda_{r e g}^{u p}$ & $\mathrm{p}=\lambda_{r e g}^{d n}$ \\
\hline \hline
\end{tabular}

The model is quite similar to SIP-SR in that the SO computes the Net Imbalance Volume (NIV), i.e. the net amount of upand down- regulation called in the trading period, then, for that net amount, it computes the marginal offer price or bid price, but, in this case, without any reversions to the spot price. The motivation is to make it easier to hedge imbalance prices by market participants, especially vertically integrated companies that may be out of balance in opposite directions in their generation and retail accounts, and to go further than SIP-SR by actually encouraging participants to help to balance the system. Apart from GB, single imbalance pricing exists in Belgium, Austria and Germany subject to various conditions. In this section, we have presented the mathematical formulation of our proposed concept applied to three imbalance pricing models. We explain and discuss the dual imbalance pricing (DIP) model, a benchmark model corresponding to the DIP model, single imbalance pricing with spot reversion (SIPSR) model, a benchmark model for the SIP-SR model, and the single imbalance pricing (SIP) model. Our concept of SO clearing the balancing market by considering the imbalances 
TABLE IV

INPUT PARAMETER DATA FOR FIVE-GENERATOR SYSTEM IN ALL MODELS

\begin{tabular}{llllll}
\hline \hline $\begin{array}{l}\text { gen } \\
\text { no. }\end{array}$ & $\begin{array}{l}\lambda^{u p} \\
(\$ / M W h)\end{array}$ & $\begin{array}{l}\lambda^{d n} \\
(\$ / M W h)\end{array}$ & $\begin{array}{l}g^{u p} \\
(M W h)\end{array}$ & $\begin{array}{l}g^{d n} \\
(M W h)\end{array}$ & $\begin{array}{l}g^{s} \\
(M W h)\end{array}$ \\
\hline$G_{1}$ & 10 & 8 & 30 & 20 & 400 \\
\hline$G_{2}$ & 11 & 7 & 40 & 40 & 500 \\
\hline$G_{3}$ & 12 & 6 & 25 & 15 & 350 \\
\hline$G_{4}$ & 13 & 5 & 35 & 25 & 450 \\
\hline$G_{5}$ & 14 & 4 & 45 & 35 & 550 \\
\hline \hline
\end{tabular}

of the generators has been applied to different imbalance pricing models. A CVaR constraint has been introduced to the optimization model in order to account for surplus generation or unmet demand, with an appropriate small probability. The resulting non-linear optimization models have been linearized through Big-M method.

\section{Proposed Dynamic DEnSity estimation AND FORECASTING USING GAMLSS}

To be implemented in practice, the above formulations require probabilistic forecasting of imbalances and we adopt a Generalized Additive Model for Location, Scale and Shape (GAMLSS) as effectively used in this context by [36]. In GAMLSS, one of a variety of density functions can be selected in which the mean and higher moments can be specified dynamically as conditional upon exogenous driving variables, [37]. In our proposed stochastic MILP models 21] and 277, the metered generation is the uncertain variable and the estimation flowchart is shown in Fig. 5. First, we fit the best distribution to the uncertain metered generation using the Akaike Information Criterion (AIC). The moments of the selected distribution are linearly regressed on selected explanatory variables (see Section VII for more details). With forecasts for the explanatory variables, the moments of the selected distribution are estimated. This distribution is then approximated using a set of scenarios [38].

\section{ILLUSTRATIVE EXAMPLES}

We assume five generators $G_{1}$ to $G_{5}$ participate in our proposed balancing market to meet the system demand. Table IV shows the values of input parameter for the five-generator system in all the three proposed models. The simulations were performed by setting the values of $\Xi^{(1)}, \Xi^{(2)}, \Xi^{(3)}, \Xi^{(4)}, \Xi^{(5)}$, and $\Xi^{(6)}$ equal to 2000 in optimization models 21 and 27 . The generation of $G_{1}, G_{2}, G_{3}, G_{4}$, and $G_{5}$ are distributed according to normal distribution ${ }^{4} \mathrm{~N}(400,0), \mathrm{N}(500,4), \mathrm{N}(350$, $8), \mathrm{N}(450,12)$ and $\mathrm{N}(550,16)$, respectively. Using these normal distributions, 1000 scenarios are sampled and then reduced to 10 scenarios presented in Fig. 6. The MILPs for all the three proposed models are coded in GAMS and solved using CPLEX solver [39]. Simulations are carried out on a computer with $16 \mathrm{~GB}$ of RAM and $1.2 \mathrm{GHz} \mathrm{CPU}$. The computational times for the DIP, SIP-SR and SIP models were 0.25 seconds, 1.69 seconds and 67.89 seconds, respectively. As observed,

\footnotetext{
${ }^{4}$ The notation $N(\mu, \sigma)$ represents the normal probability distribution function (PDF) with mean $\mu$ and standard deviation $\sigma$.
}

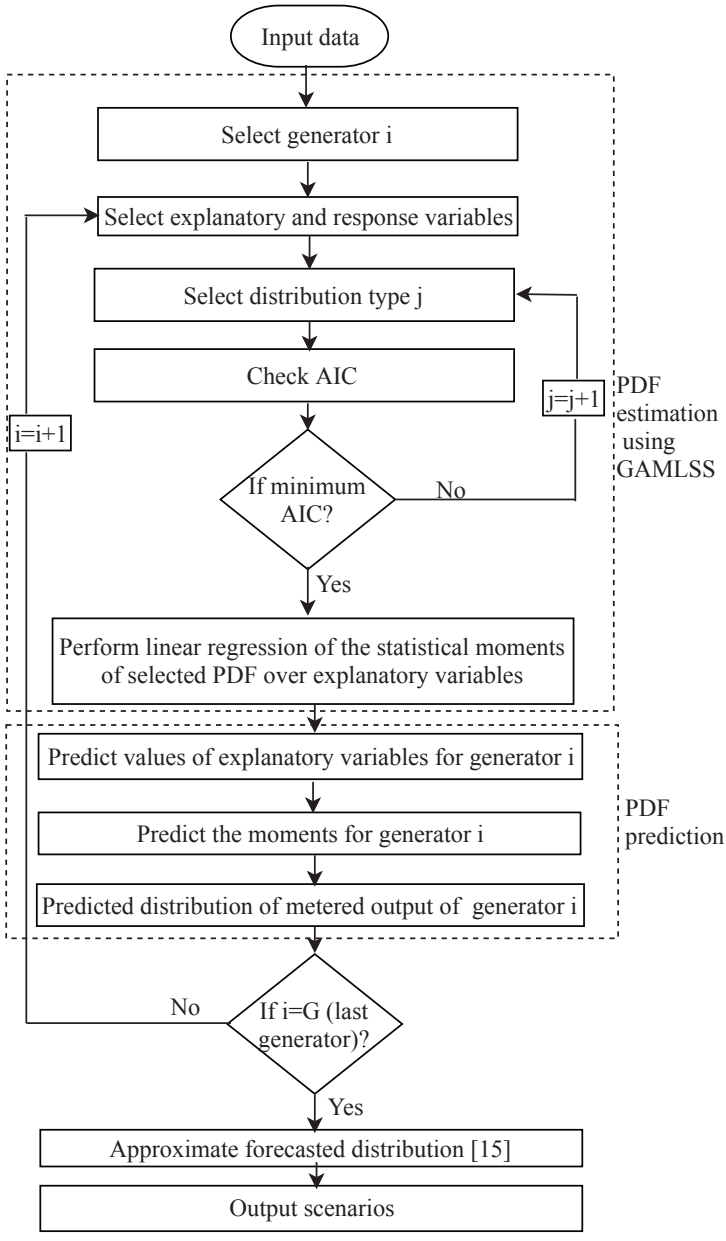

Fig. 5. Estimation and forecasting the dynamic distribution of metered generation $g_{i}^{m}$ using GAMLSS

the computational time for the SIP model is the highest due to the several binary variables introduced for the linearization process 5 . We can reduce the computational time in our MILP models in two ways: first, we can employ decomposition algorithms to break the MILP model into smaller and easierto-solve optimization problems [40], [41]. Second, we can use scenario generation and reduction algorithm to reduce the size of our MILP models [42], [43]. Using the reduced scenarios with updated probabilities that can represent the entire data set enables us to reduce the computational time significantly, which is desirable for real-time dispatch decisions. We employ the second approach in our paper and through a proposed scenario generation and reduction algorithm, the number of scenarios in our MILP models is reduced to 10 scenarios with updated probabilities. We select generator $G_{1}$ (the first generator in the merit-order dispatch) as the (renewable-) intermittent generator participating in the balancing market. The standard deviation of output generation for $G_{1}$ is changed from $\sigma_{\mathrm{G} 1}=0 \mathrm{MWh}$ (firm output) to $\sigma_{\mathrm{G}_{1}}=70 \mathrm{MWh}$ (intermittent output). As seen in Fig. 7, the BSC increases with increasing output intermittency of $G_{1}$ in SIP and SIP-SR

\footnotetext{
${ }^{5}$ It is not the primary focus of the paper to reduce the computation time. However, it could be an interesting future work to look at possible ways to reduce the computation time for the SIP model.
} 


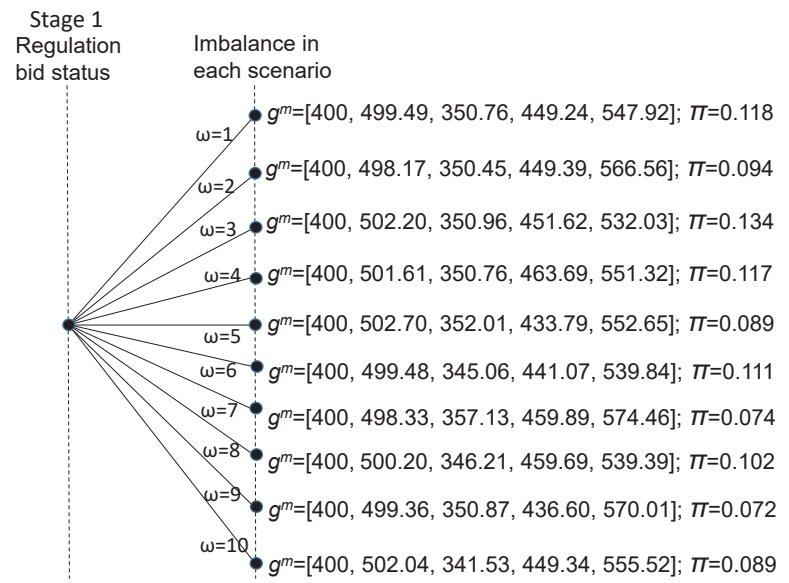

Fig. 6. Sample scenario tree for one-stage stochastic MILPs for all the three models

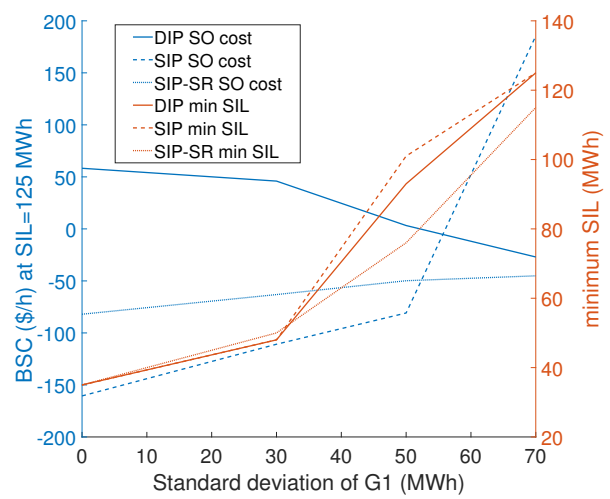

Fig. 7. Effect of varying standard deviation of $G_{1}$ metered generation on balancing and settlement cost (BSC) and minimum $S I L$ for five-generator system (BSC axis is scaled down by 10 for SIP model)

models. This indicates that these models do not prefer to stay with the cheaper generator $G_{1}$ as it becomes more intermittent. Both SIP and SIP-SR use marginal cost pricing, and in this case they provide strong signals on the cost of intermittency. The DIP model however, being based on average pricing, is not able to give this signal as the BSC reduces in its case with the increase in intermittency of $G_{1}$. This is one of the reasons why the GB market moved from DIP to SIP model.

The effect of probabilistic constraint (10) is also shown in Fig. 7 The SO can satisfy the energy-balance constraint in $90 \%$ of scenarios $(\beta=0.9)$ with lower SIL level when the $\sigma_{\mathbf{G} \mathbf{1}}$ is low. However, when generator $G 1$ becomes more intermittent (higher $\sigma_{\mathbf{G} 1}$ ), the SO needs to tolerate higher SIL levels in order to satisfy the probabilistic constraint 10 in $90 \%$ of scenarios.

\section{A. Comparison of the proposed optimal-dispatch models with the benchmark models}

In the benchmark model, the recourse costs incurred by the SO decision in the BMC (term $\mathbb{E}$ (ISC) in optimization model (12)) are ignored. This benchmark optimal-dispatch model is solved and the BMC is calculated. The ISC for each scenario is also calculated and weighted average of the imbalance costs $\mathbb{E}$ (ISC) is obtained. Results in Table $\mathrm{V}$ s show the predictive nature of our optimal-dispatch model. Under $\sigma_{\mathrm{G} 1}=30 \mathrm{MWh}$, the Bench-DIP model leads to $\mathrm{BSC}=226.6 \$ / h$. This is while
TABLE $\mathrm{V}$

COMPARISON OF BMC, ISC AND BSC (ALL VALUES ARE IN $\$ / h$ ) FOR THE BENCHMARK (BENCH.) AND PROPOSED (PROP.) MODELS FOR $\beta=0.9$ AND SIL $=125 M W h$ AT DIFFERENT STANDARD DEVIATION (STD. DEV.) OF $G_{1}$

\begin{tabular}{l|l|ll|ll|ll}
\hline \hline $\begin{array}{l}\text { std } \\
\text { der }\end{array}$ & & $\begin{array}{l}\text { DIP } \\
\text { Bench. }\end{array}$ & Prop. & $\begin{array}{l}\text { SIP- } \\
\text { SR } \\
\text { Bench. }\end{array}$ & Prop. & $\begin{array}{l}\text { SIP } \\
\text { Bench. }\end{array}$ & Prop. \\
\hline 0 & BMC & -55 & 210 & -55 & 230 & -55 & 1365 \\
& ISC & 216.2 & -151.7 & 274.0 & -1390.5 & 274.0 & -2185 \\
& BSC & 161.1 & 58.2 & 219.0 & -160.5 & 219.0 & -820 \\
\hline 30 & BMC & -75 & 210 & -75 & 930 & -75 & 1035 \\
& ISC & 301.6 & -164 & 445.2 & -1040.8 & 445.2 & -1666 \\
& BSC & 226.6 & 46 & 370.2 & -110.8 & 370.2 & -631 \\
\hline 50 & BMC & -195 & 210 & -195 & 600 & -195 & 735 \\
& ISC & 295.8 & -206.7 & 338.8 & -680.8 & 342.4 & -1233 \\
& BSC & 100.8 & 3.2 & 143.8 & -80.8 & 147.4 & -498 \\
\hline \hline
\end{tabular}

under our Prop-DIP model, the BSC cost is reduced to $46 \$ / h$. Under benchmark model, the SO has a revenue of $\mathrm{BMC}=75 \$ / h$ in the balancing market, however, the recourse cost of its decision amounts to $\mathrm{ISC}=301.6 \$ / h$. This is while in our proposed optimal-dispatch model, the SO takes into account the ISC in the BM decision making process. The BMC is increased to $140 \$ / M W h$ at the benefit of reduced ISC to $164 \$ / M W h$ revenue. Once we increase the intermittency level of $G 1\left(\sigma_{\mathbf{G} 1}=50 M W h\right)$, the same pattern is observed. The benchmark optimal-dispatch model ignores the ISC leading to $100.8 \$ / h$ for total system cost (BSC). But in our proposed model, the BSC is reduced in order of five to $\mathrm{BSC}=3.2 \$ / h$ at $\mathrm{SIL}=125 \mathrm{MWh}$. The comparative analysis of prop-SIP-SR and prop-SIP models with their corresponding benchmark models show similar pattern for the costs. For our case studies, the Prop-SIP model has the lowest BSC as compared to the Prop-DIP and Prop-SIP-SR models.

\section{NUMERICAL RESULTS}

In this section, we assume eight generator ${ }^{6}$ participating in the BM, where each generator represents one area in the Nordic electricity market7 The metered generation of these generators are taken from Nord Pool website [46].

\section{A. Proposed GAMLSS-based scenario generation algorithm in} Fig. 5

To estimate and forecast, the dynamic distribution of metered generations, the GAMLSS packages in $\mathrm{R}$ is used [47] and this linearly regresses the parameters of the assumed distribution on lagged values, wind generation, hydropower and demand. Table VI gives the value of Akaike Information Criterion (AIC) for different distributions for the data of $G_{1}$ metered generation. The skew $\mathrm{t}$ distribution was found to have minimum AIC. Similarly, the analysis was performed for other generators in the system. Once the skew $t$ distribution

${ }^{6}$ With eight generators it was easier to interpret the choice of generators by the system operator (SO) for balancing market considering their intermittency, which is reflected by the ISC. Therefore, the choice of five- and eightgenerator systems was for the ease of demonstration. As all our proposed models have been linearized, decomposition methods can be implemented to study a real system [38], [40], [41], [44], [45].

${ }^{7}$ Out of the eight generators, three of them are located in Sweden, one in Denmark and four in Norway. 
TABLE VI

AIC VALUES FOR DIFFERENT DISTRIBUTIONS TESTED ON $G_{1}$ METERED GENERATION

\begin{tabular}{llllll}
\hline \hline Distribution & Skew t & $\begin{array}{l}\text { Log } \\
\text { normal }\end{array}$ & $\begin{array}{l}\text { Negative } \\
\text { binomial }\end{array}$ & Normal & Poisson \\
\hline AIC values & 4000 & 4894.05 & 4901.03 & 4925.47 & 23262.09 \\
\hline \hline
\end{tabular}

TABLE VII

INTERCEPTS AND COEFFICIENTS OBTAINED FROM GAMLSS FOR THE GENERATORS $G_{1}$ TO $G_{4}$

\begin{tabular}{llllllll}
\hline \hline & $\alpha_{1}$ & $\gamma_{1}$ & $\gamma_{2}$ & $\gamma_{3}$ & $\beta_{1}$ & $\beta_{2}$ & $\beta_{3}$ \\
\hline$G_{1}$ & 35.6 & 0.072 & 0.091 & 0.424 & 0.451 & 0.194 & 5.81 \\
\hline$G_{2}$ & 661.1 & 0.057 & 0.061 & 0.338 & 0.551 & 0.468 & 11.58 \\
\hline$G_{3}$ & 716.6 & 0.045 & 0.009 & 0.431 & 1.976 & 0.450 & -33.05 \\
\hline$G_{4}$ & -774.8 & 0.115 & 0.052 & 0.380 & 0.273 & 0.828 & \\
\hline \hline
\end{tabular}

is selected for $G_{1}$ metered generation, different parameters of this distribution (mean, standard deviation, skewness and kurtosis) are linearly regressed on those explanatory variables which were significant at 5\%. The linear expression 28 shows the regression of the mean value $\left(\mu_{t}^{G_{1}}\right)$ for $G_{1}$ metered generation over its explanatory variables. In (28), $\alpha_{1}$ indicates the intercept of the response variable $\mu^{G_{1}}$. The $\gamma_{1}, \gamma_{2}$ and $\gamma_{3}$ are the coefficients of the lag values of $\mu_{t}^{G_{1}}$. The coefficients corresponding to wind generation $\left(\right.$ wind $\left._{t}\right)$, demand $\left(\right.$ dem $\left._{t}\right)$ and hydropower $\left(h y d_{t}\right)$ are $\beta_{1}, \beta_{2}$ and $\beta_{3}$, respectively.

$$
\begin{aligned}
\mu_{t}^{G_{1}}= & \alpha_{1}+\gamma_{1} \mu_{t-1}^{G_{1}}+\gamma_{2} \mu_{t-2}^{G_{1}}+\gamma_{3} \mu_{t-3}^{G_{1}}+\beta_{1} \operatorname{wind}_{t}^{G_{1}} \\
& +\beta_{2} \operatorname{dem}_{t}^{G_{1}}+\beta_{3} h y d_{t}^{G_{1}}
\end{aligned}
$$

Tables VII and VIII give the values of intercepts and coefficients for the mean value of metered generation as obtained from GAMLSS. Table IX shows the values of mean $(\mu)$, standard deviation $(\sigma)$, skewness $(\nu)$ and kurtosis $(\tau)$ of the metered generation calculated by GAMLSS. These values are used for scenario generation from the skew $t$ distribution. Each

TABLE VIII

INTERCEPTS AND COEFFICIENTS OBTAINED FROM GAMLSS FOR THE GENERATORS $G_{5}$ TO $G_{8}$

\begin{tabular}{llllllll}
\hline \hline & $\alpha_{1}$ & $\gamma_{1}$ & $\gamma_{2}$ & $\gamma_{3}$ & $\gamma_{4}$ & $\beta_{2}$ & $\beta_{3}$ \\
\hline$G_{5}$ & -21.5 & 0.003 & 0 & -0.006 & 1.086 & -0.034 & -1.38 \\
\hline$G_{6}$ & 687.7 & 0 & 0 & -0.004 & 0.816 & 0.199 & -7.83 \\
\hline$G_{7}$ & 6287 & -0.003 & -0.015 & -0.004 & -0.981 & 0.5346 & -39.13 \\
\hline$G_{8}$ & 11090 & 0.003 & -0.722 & -0.254 & -0.564 & -2.341 & 41.35 \\
\hline \hline
\end{tabular}

TABLE IX

OBTAINED VALUES OF MEAN, STANDARD DEVIATION, SKEWNESS AND KURTOSIS FOR THE GENERATORS FROM GAMLSS

\begin{tabular}{lllll}
\hline \hline & $\mu(\mathrm{MWh})$ & $\sigma(\mathrm{MWh})$ & $\nu$ & $\tau$ \\
\hline$G_{1}$ & 1269.2 & 266.24 & 0.6082 & 5.6916 \\
\hline$G_{2}$ & 2560.7 & 494.664 & 0.8782 & 144.459 \\
\hline$G_{3}$ & 10988 & 236.78 & -0.1696 & 3.309 \\
\hline$G_{4}$ & 1488.8 & 209.22 & 0.6236 & 12.114 \\
\hline$G_{5}$ & 1523.8 & 0 & 1.8538 & 8.6119 \\
\hline$G_{6}$ & 6749.8 & 0.0433 & -0.2543 & 0.072 \\
\hline$G_{7}$ & 3214.1 & 0 & 0.8149 & 3.6506 \\
\hline$G_{8}$ & 3562.2 & 0 & 1.3201 & 3.4879 \\
\hline \hline
\end{tabular}

TABLE $X$

INPUT PARAMETER DATA FOR EIGHT-GENERATOR SYSTEM IN DIP, SIP-SR AND SIP MODELS

\begin{tabular}{llllll}
\hline \hline Gen & $\begin{array}{l}\lambda^{u p} \\
(\$ / M W h)\end{array}$ & $\begin{array}{l}\lambda^{d n} \\
(\$ / M W h)\end{array}$ & $\begin{array}{l}g^{u p} \\
(M W h)\end{array}$ & $\begin{array}{l}g^{d n} \\
(M W h)\end{array}$ & $\begin{array}{l}g^{s} \\
(M W h)\end{array}$ \\
\hline$G_{1}$ & 15 & 4 & 300 & 300 & 1404.9 \\
\hline$G_{2}$ & 16 & 5 & 200 & 200 & 2700.2 \\
\hline$G_{3}$ & 17 & 6 & 1100 & 1100 & 11405 \\
\hline$G_{4}$ & 18 & 7 & 100 & 100 & 1604.7 \\
\hline$G_{5}$ & 19 & 8 & 100 & 100 & 1523.8 \\
\hline$G_{6}$ & 20 & 9 & 700 & 700 & 7122 \\
\hline$G_{7}$ & 21 & 10 & 200 & 200 & 3214.1 \\
\hline$G_{8}$ & 22 & 11 & 200 & 200 & 3562.2 \\
\hline \hline
\end{tabular}

TABLE XI

COMPARISON OF STATUS OF UP- AND DOWN-REGULATION BIDS FOR THE EIGHT-GENERATOR SYSTEM

\begin{tabular}{llllllllll}
\hline \hline Gen & & $G_{1}$ & $G_{2}$ & $G_{3}$ & $G_{4}$ & $G_{5}$ & $G_{6}$ & $G_{7}$ & $G_{8}$ \\
\hline DIP & $\phi^{u p}$ & $1(0)$ & $0(0)$ & $0(0)$ & $0(1)$ & $0(0)$ & $0(0)$ & $0(0)$ & $0(0)$ \\
Prop(Ben) & $\phi^{d n}$ & $0(1)$ & $0(0)$ & $0(0)$ & $0(0)$ & $0(1)$ & $1(0)$ & $0(1)$ & $0(1)$ \\
\hline SIP-SR & $\phi^{u p}$ & $0(0)$ & $1(0)$ & $0(0)$ & $0(1)$ & $0(0)$ & $0(0)$ & $0(0)$ & $0(0)$ \\
Prop(Ben) & $\phi^{d n}$ & $0(1)$ & $0(0)$ & $0(0)$ & $1(0)$ & $1(1)$ & $0(0)$ & $1(1)$ & $1(1)$ \\
\hline SIP & $\phi^{u p}$ & $0(0)$ & $0(0)$ & $0(0)$ & $0(1)$ & $0(0)$ & $0(0)$ & $0(0)$ & $0(0)$ \\
Prop(Ben) & $\phi^{d n}$ & $0(1)$ & $0(0)$ & $0(0)$ & $0(0)$ & $1(1)$ & $0(0)$ & $1(1)$ & $1(1)$ \\
\hline \hline
\end{tabular}

skew $\mathrm{t}$ distribution is sampled by 1000 scenarios which are given as input to the fast forward selection reduction algorithm [38]. As a result of the scenario reduction algorithm, ten scenarios are obtained along with their updated probabilities, which are then used in the proposed and benchmark model 8 The computational time for eight-generator system turned out to be 0.11 seconds, 1.5 seconds and 840.41 seconds for the DIP, SIP-SR and SIP models respectively. Again, the computational time for the SIP model was the highest even for this case study due to the several binary variables introduced for the linearization process.

\section{B. Proposed economic-dispatch models}

Table XI shows the status of up- and down-regulation offers and bids for the eight generators when the same input is given to all the proposed and benchmark models. The minimum SIL for DIP and SIP-SR was $603 M W h$ while for SIP it was $435 M W h$ to satisfy the probabilistic energy-balance constraint $(10)$ at $\beta=0.9$. It can be seen that some of the generators up-regulate while none of them down-regulate for

TABLE XII

COMPARISON OF BSC, BMC AND ISC (ALL VALUES ARE IN $\$ / h$ ) FOR THE BENCHMARK (BENCH.) AND PROPOSED (PROP.) MODELS (POSITIVE VALUE INDICATES COST AND NEGATIVE VALUE IS REVENUE)

\begin{tabular}{lllllll}
\hline \hline \multicolumn{3}{c}{ DIP model } & \multicolumn{2}{l}{ SIP-SR model } & \multicolumn{2}{l}{ SIP model } \\
\hline & Bench. & Prop. & Bench. & Prop. & Bench. & Prop. \\
\hline BMC & -4400 & -1800 & -4400 & -2500 & -4400 & -4200 \\
\hline ISC & 69.4 & -3671.3 & 656.7 & -3809.4 & 745.7 & -3018.6 \\
\hline BSC & -4330.5 & -5471.3 & -3743.2 & -6309.4 & -3654.2 & -7218.6 \\
\hline \hline
\end{tabular}

\footnotetext{
${ }^{8} \mathrm{We}$ use the same data for all the three dispatch models, since the generator characteristics can be taken as representative across both the regions (Nordic countries and Great Britain).
} 
the given set of inputs. As shown in Table $\mathrm{X}, G_{1}$ is the most expensive for down-regulation and has a high standard deviation (see Table IX), hence it was not chosen in any of the proposed models due to its high intermittency level. However, it was chosen by all benchmark models which ignore the intermittency cost of $G_{1}$. Table XII clearly shows the predictive nature of our optimal-dispatch model. The BenchDIP model results in $\mathrm{BSC}=-4330.5 \$ / h$ while our PropDIP model gives $\mathrm{BSC}=-5471.3 \$ / h$. This indicates a higher revenue in our Prop-DIP model as compared to the BenchDIP model. The results followed the trend as observed in the Illustrative examples (see Table $\mathrm{V}$ ). Even though the SO earns a higher revenue $(\mathrm{BMC}=4400 \$ / h$ ) in Bench-DIP model, the recourse cost of its decision leads to ISC of $69.4 \$ / h$. In our Prop-DIP model however, the ISC $=-3671.3 \$ / h$. This results in a net reduction in BSC by $1140.8 \$ / h$ in our Prop-DIP model as compared to Bench-DIP model. This reduction in BSC can be accounted to the fact that in our Prop-DIP model, the objective function of the optimization problem comprises of both the BMC and ISC (as given in equation (11)) that are minimized. However, in the benchmark model, only the BMC is minimized and the output for binary variables $\phi_{i}^{d n}$ and $\phi_{i}^{u p}$ from minimizing BMC serves as inputs to $11 \mathrm{~b}$ and (11c) respectively for finding out the ISC. This value of ISC for the benchmark model turns out to be higher than that in our proposed model as the intermittency of the generators do not play any role in selecting the generators to be operated in the balancing market. Another interesting point to explain the large differences in the ISC values of Table XII as compared to those in Table $\mathrm{V}$ would be that it has to do with the differences in the input values of $\lambda^{u p}, \lambda^{d n}, g^{u p}, g^{d n}$ and $g^{s}$ in the fiveand eight-generator systems. Table IV and Table $\mathrm{X}$ give the values of the inputs considered for the $\lambda^{u p}, \lambda^{d n}, g^{u p}, g^{d n}$ and $g^{s}$ in our simulations for five- and eight-generator systems, respectively. In order to obtain values for the scheduled output of the generation, $g^{s}$, we take average of the 10 values of $g_{i, \omega}^{m}$ that were obtained from the proposed scenario generation algorithm based on predictive conditional dynamic density distributions. The study has been performed on real market data acquired from the Nord Pool website to find the values for $g_{i, \omega}^{m}$. As the magnitudes of all these input values for the eight-generator system in the Table $\mathrm{X}$ are larger as compared to those assumed for the five-generator system in Table IV. the results of the ISCs have larger differences in Table XII as compared to those in Table $\mathrm{V}$.

The reduction in BSC for the Prop-SIP-SR and Prop-SIP models were $2566.2 \$ / h$ and $3564.4 \$ / h$ respectively as compared to the corresponding benchmark models. A similar trend of reduction in BSC in the proposed models of Numerical examples has reaffirmed our observations from the Illustrative examples. The less reduction in BSC for the Prop-DIP-model can be accounted for in Table $\mathrm{X}$. It can be seen that $G_{1}$ was chosen by the Prop-DIP model for up-regulation as it was the cheapest for up-regulation but also more variable than $G_{4}$, which was chosen by the corresponding Bench-DIP model.

\section{CONCLUSION}

This paper proposes three stochastic optimization models in order to enable the SO to dispatch into the balancing market not just based on the marginal cost but also on the intermittency of the generators. This capability will become increasingly important as more intermittent and stochastic generators come on to the system and indeed wish to take part in balancing actions. The three one-stage stochastic optimization models are based on different imbalance pricing methods. Out of the three proposed models, the single imbalance price models are able to give a clear signal to the stochastic (cheaper but more uncertain) generators to invest in technologies or hedge efficiently if they want to be chosen in the balancing market by the SO. For the same inputs to the MILP models, SIP model gives more saving for the $\mathrm{SO}$ as compared to the DIP model. Therefore, we observe why it was beneficial for the GB electricity market to move from DIP model to SIP model in 2015.

\section{FURTHER DISCUSSIONS AND FUTURE WORK}

In Nordic market, the balancing market is a two-stage market. In the first stage, the transmission constraints are ignored and the dispatching is done only based on the energy imbalances, then if the re-dispatch in the first stage is violating some transmission constraints, in the second stage, the counter trading will be performed [28]. The balancing market in GB is explicitly designed to deal with energy imbalances and any actions, which the system operator takes for locational or voltage reasons are recorded separately and included in the recovery of overall system costs by the SO. They are not part of the balancing market [30]. Thus, from a practical view-point while activating the bids in the balancing market, transmission constraints are not considered in the first stage of Nordic balancing market and GB electricity market. As our paper primarily focuses on these markets, we do not model transmission constraints in our paper. However, the explicit modeling of transmission constraints in our proposed model is a good future extension of our work, which adjusts our model for other electricity markets too.

Reference [48] explains how the SO in Sweden procures losses before the DA market clearing. In the balancing market, the SO activates the bids based on the merit order list independent of how large losses might occur due to activating a particular bid [29]. Later, the SO pays for the transmission grid losses, which it has to buy before the DA market clearing. Losses in GB are included in the settlement system, not the balancing market. All contracts are settled in comparison with metered volumes and imbalance volumes after the delivery periods and zonal loss factors are applied at that stage [49]. But in some other electricity markets, if it is important to model losses then the modeling of ohmic losses in our proposed formulation can be considered as a good future extension of our work.

The tests performed in Sections VI and VII serve the purpose of demonstrating the functionality of the proposed models. While the existing work tests the proposed models with fiveand eight-generator systems, it is desirable to apply these models to a full system [38], [40], [41], [44], [45]. This will 
be a further extension of the existing work.

The issue of fairness and openness in scenario generation and reduction, as employed in our method, is not addressed in the literature. This is one promising direction of future research.

\section{ACKNOWLEDGEMEnT}

Dr. Date acknowledges useful discussions about the GB electricity market with Dr A. H. Alikhanzadeh. The authors also acknowledge useful comments regarding the role of wind energy in the UK balancing market from Jean Hamman and Martin Bradley, both from National Grid UK. We are also grateful to Robert Eriksson from Svenska kraftnät (Swedish Transmission System Operator) for answering our questions about the Nordic balancing market.

\section{REFERENCES}

[1] IEA. (2018) World Energy Outlook 2018. [Online]. Available: https://www.iea.org/weo2018/

[2] eurostat. (2019) Renewable energy statistics. [Online]. Available: https://ec.europa.eu/eurostat/statistics-explained/index.php/Renewable_ energy_statistics\#Renewable_energy_produced_in_the_EU_increased_ by_two_thirds_in_2007-2017

[3] E. Department for Business and I. Strategy. (2018) UK Energy Statistics, Q3 2018. [Online]. Available: https://assets.publishing.service.gov.uk/government/uploads/system/ uploads/attachment_data/file/766776/Press_Notice_December_2018.pdf

[4] EIA. (2019) U.S. renewable electricity generation has doubled since 2008. [Online]. Available: https://www.eia.gov/todayinenergy/detail. php?id=38752

[5] IEA. (2014) The Power of Transformation. [Online]. Available: https://webstore.iea.org/the-power-of-transformation

[6] P. Sorknæs, A. N. Andersen, J. Tang, and S. Strøm, "Market integration of wind power in electricity system balancing," Energy Strategy Reviews, vol. 1, no. 3, pp. 174-180, 2013.

[7] H. Lund, "Electric grid stability and the design of sustainable energy systems," International Journal of Sustainable Energy, vol. 24, no. 1, pp. 45-54, 2005.

[8] "European parliament, council, directive 2003/54/ ec of the european parliament and of the council of 26 june 2003 concerning common rules for the internal market in electricity and repealing directive 96/92/ec e statements made with regard to decommissioning and waste management activities," pp. 37-56, 2003

[9] "National grid, managing intermittent and inflexible generation in the balancing mechanism, national grid," Sept. 2011.

[10] EWEA. (2015) Balancing responsibility and costs of wind power plants. [Online]. Available: http: //www.ewea.org/fileadmin/files/library/publications/position-papers/ EWEA-position-paper-balancing-responsibility-and-costs.pdf

[11] L. Vandezande, L. Meeus, R. Belmans, M. Saguan, and J.-M. Glachant, "Well-functioning balancing markets: A prerequisite for wind power integration," Energy Policy, vol. 38, no. 7, pp. 3146-3154, 2010.

[12] Y. Guo, Y. Gong, Y. Fang, and P. P. Khargonekar, "Stochastic minimization of imbalance cost for a virtual power plant in electricity markets," in ISGT 2014, Feb 2014, pp. 1-5.

[13] J. Liang, S. Grijalva, and R. G. Harley, "Increased wind revenue and system security by trading wind power in energy and regulation reserve markets," IEEE Transactions on Sustainable Energy, vol. 2, no. 3, pp. 340-347, July 2011

[14] A. Hellmers, M. Zugno, A. Skajaa, and J. M. Morales, "Operational strategies for a portfolio of wind farms and chp plants in a two-price balancing market," IEEE Transactions on Power Systems, vol. 31, no. 3, pp. 2182-2191, May 2016.

[15] J. Matevosyan and L. Soder, "Minimization of imbalance cost trading wind power on the short-term power market," IEEE Transactions on Power Systems, vol. 21, no. 3, pp. 1396-1404, 2006.

[16] P. M. S. Frade, J. P. S. Catalão, J. P. Pereira, and J. J. E. Santana, "Balancing reserves in a power system with high wind penetration - evidence from portugal," in 15th International Conference on the European Energy Market (EEM), June 2018, pp. 1-4.
[17] A. G. Vlachos and P. N. Biskas, "Demand response in a real-time balancing market clearing with pay-as-bid pricing," IEEE Transactions on Smart Grid, vol. 4, no. 4, pp. 1966-1975, Dec 2013.

[18] A. Helander, H. Holttinen, and J. Paatero, "Impact of wind power on the power system imbalances in Finland," IET renewable power generation, vol. 4, no. 1, pp. 75-84, 2010.

[19] J. Browell, "Risk constrained trading strategies for stochastic generation with a single-price balancing market," Energies, vol. 11, no. 6, p. 1345 , 2018.

[20] R. A. van der Veen and R. A. Hakvoort, "The electricity balancing market: Exploring the design challenge," Utilities Policy, vol. 43, pp. 186-194, 2016

[21] T. Brijs, K. De Vos, C. De Jonghe, and R. Belmans, "Statistical analysis of negative prices in european balancing markets," Renewable Energy, vol. 80, pp. 53-60, 2015.

[22] L. Hirth and I. Ziegenhagen, "Balancing power and variable renewables: Three links," Renewable and Sustainable Energy Reviews, vol. 50, pp. 1035-1051, 2015.

[23] J. P. Chaves-Ávila, R. A. Hakvoort, and A. Ramos, "The impact of european balancing rules on wind power economics and on short-term bidding strategies," Energy Policy, vol. 68, pp. 383-393, 2014.

[24] C. Fernandes, P. Frías, and J. Reneses, "Participation of intermittent renewable generators in balancing mechanisms: A closer look into the spanish market design," Renewable Energy, vol. 89, pp. 305-316, 2016.

[25] P. Pinson, C. Chevallier, and G. N. Kariniotakis, "Trading wind generation from short-term probabilistic forecasts of wind power," IEEE Transactions on Power Systems, vol. 22, no. 3, pp. 1148-1156, 2007.

[26] A. Skajaa, K. Edlund, and J. M. Morales, "Intraday trading of wind energy," IEEE Transactions on Power Systems, vol. 30, no. 6, pp. 3181$3189,2015$.

[27] S. Kraftnät. (2019) Overview of the Requirements on Reserves. [Online]. Available: https://www.svk.se/siteassets/aktorsportalen/elmarknad/ information-om-reserver/reserve-markets.pdf

[28] ENTSOE. (2016) Nordic Balancing Philosophy. [Online]. Available: https://docstore.entsoe.eu/Documents/Publications/SOC/Nordic/ Nordic_Balancing_Philosophy_160616_Final_external.pdf

[29] S. Kraftnät, "The swedish electricity market and the role of svenska kraftnät," Published Jan, 2007. [Online]. Available: https://inis.iaea.org/ collection/NCLCollectionStore/_Public/42/022/42022239.pdf

[30] E. M. A. Team. (2019) System Prices Analysis Report. [Online]. Available: https://www.elexon.co.uk/about/key-data-reports/ system-prices-analysis-report/

[31] nationalgridESO. (2020) Short-term operating reserve (STOR). [Online]. Available: https://www.nationalgrideso.com/balancing-services/ reserve-services/short-term-operating-reserve-stor

[32] PJM. (2019) About PJM. [Online]. Available: https://www.pjm.com/ about-pjm/who-we-are.aspx

[33] (2019) Understanding the Differences Between PJM's Markets. [Online]. Available: https://learn.pjm.com/-/media/about-pjm/newsroom/fact-sheets/ understanding-the-difference-between-pjms-markets-fact-sheet.ashx? la=en

[34] A. L. S. Mendes, N. de Castro, R. Brandão, L. Câmara, and M. Moszkowicz, "The role of imbalance settlement mechanisms in electricity markets: A comparative analysis between uk and brazil," in 13th International Conference on the European Energy Market (EEM), June 2016, pp. 1-6.

[35] R. T. Rockafellar, S. Uryasev et al., "Optimization of conditional valueat-risk," Journal of risk, vol. 2, pp. 21-42, 2000.

[36] A. Bello, D. W. Bunn, J. Reneses, and A. Muñoz, "Medium-term probabilistic forecasting of electricity prices: A hybrid approach," IEEE Transactions on Power Systems, vol. 32, no. 1, pp. 334-343, Jan 2017.

[37] D. M. Stasinopoulos, R. A. Rigby et al., "Generalized additive models for location scale and shape (gamlss) in r," Journal of Statistical Software, vol. 23, no. 7, pp. 1-46, 2007.

[38] A. J. Conejo, M. Carrión, J. M. Morales et al., Decision making under uncertainty in electricity markets. Springer, 2010, vol. 1.

[39] IBM, "V12. 1: User's manual for cplex," International Business Machines Corporation, vol. 46, no. 53, p. 157, 2009.

[40] E. Moiseeva and M. R. Hesamzadeh, "Strategic bidding of a hydropower producer under uncertainty: Modified benders approach," IEEE Transactions on Power Systems, vol. 33, no. 1, pp. 861-873, Jan 2018.

[41] Y. Tohidi, M. R. Hesamzadeh, and F. Regairaz, "Modified benders decomposition for solving transmission investment game with risk measure," IEEE Transactions on Power Systems, vol. 33, no. 2, pp. 1936-1947, March 2018. 
[42] J. C. do Prado and W. Qiao, "A stochastic decision-making model for an electricity retailer with intermittent renewable energy and short-term demand response," IEEE Transactions on Smart Grid, vol. 10, no. 3, pp. 2581-2592, May 2019.

[43] J. Shen, C. Jiang, Y. Liu, and X. Wang, "A microgrid energy management system and risk management under an electricity market environment," IEEE Access, vol. 4, pp. 2349-2356, 2016.

[44] A. J. Conejo, E. Castillo, R. Minguez, and R. Garcia-Bertrand, Decomposition techniques in mathematical programming: engineering and science applications. Springer Science \& Business Media, 2006.

[45] C. Sagastizábal, "Divide to conquer: decomposition methods for energy optimization," Mathematical programming, vol. 134, no. 1, pp. 187-222, 2012.

[46] NordPool. (2017) Historical market data. [Online]. Available: https: //www.nordpoolgroup.com/historical-market-data/

[47] R. Rigby, D. Stasinopoulos, and C. Akantziliotou, "Instructions on how to use the gamlss package in r (2008)," Available at: http://www. gamlss. org/wp-content/uploads/2013/01/gamlss-manual. pdf.

[48] J. Sahlin, R. Eriksson, M. T. Ali, and M. Ghandhari, "Transmission line loss prediction based on linear regression and exchange flow modelling," in 2017 IEEE Manchester PowerTech, June 2017, pp. 1-6.

[49] ELEXON. Line Loss Factors. [Online]. Available: https://www.elexon. co.uk/operations-settlement/losses/

\section{APPENDIX}

\section{A. Linearizations related to DIP model}

The non-liear expression, $\Delta_{\omega}^{-}=\operatorname{Max}\left(-\Delta_{\omega}, 0\right)$, can be linearized by replacing it with the following two equations:

$$
\begin{aligned}
& 0 \leq \Delta_{\omega}^{-} \leq b_{\omega}^{-} \Xi^{(1)} \\
& -\Delta_{\omega} \leq \Delta_{\omega}^{-} \leq-\Delta_{\omega}+\left(1-b_{\omega}^{-}\right) \Xi^{(1)}
\end{aligned}
$$

A similar linearization has been performed for $I_{i, w}^{+}=\phi_{i}^{d n} \Delta_{\omega}^{+}$ by using the following equations:

$$
\begin{aligned}
& 0 \leq I_{i, w}^{+} \leq \phi_{i}^{d n} \Xi^{(1)} \\
& \Delta_{\omega}^{+}-\left(1-\phi_{i}^{d n}\right) \Xi^{(1)} \leq I_{i, w}^{+} \leq \Delta_{\omega}^{+}
\end{aligned}
$$

Again, for the non-linear expression, $I_{i, w}^{u p}=s_{\omega} \phi_{i}^{u p}$, it can be replaced by the equations given below:

$$
\begin{aligned}
& 0 \leq I_{i, w}^{u p} \leq \phi_{i}^{u p} \Xi^{(1)} \\
& s_{\omega}-\left(1-\phi_{i}^{u p}\right) \Xi^{(1)} \leq I_{i, w}^{u p} \leq s_{\omega}
\end{aligned}
$$

Similarly, for $I_{i, w}^{-}=\phi_{i}^{u p} \Delta_{\omega}^{-}$, the following equations can be used for linearizaion:

$$
\begin{aligned}
& 0 \leq I_{i, w}^{-} \leq \phi_{i}^{u p} \Xi^{(1)} \\
& \Delta_{\omega}^{-}-\left(1-\phi_{i}^{u p}\right) \Xi^{(1)} \leq I_{i, w}^{-} \leq \Delta_{\omega}^{-}
\end{aligned}
$$

The 'If' condition in (1c) is written as linear constraints $\sum_{i} \phi_{i}^{u p} \geq 1-\Xi^{(1)}(1-\bar{\phi})$ and $\sum_{i} \phi_{i}^{u p} \leq 1+\Xi^{(1)}(\bar{\phi})$.

The bi-linear term, $z_{\omega}^{d n}=\underline{\phi} c_{\omega}$, corresponding to 18 , can be linearized as:

$$
\begin{aligned}
& 0 \leq z_{\omega}^{d n} \leq \underline{\phi} \Xi^{(1)} \\
& c_{\omega}-(1-\underline{\phi}) \Xi^{(2)} \leq z_{\omega}^{d n} \leq c_{\omega}
\end{aligned}
$$

Similarly, another bi-linear expression, $z_{\omega}^{u p}=\bar{\phi} s_{\omega}$, in 18 can be replaced by the following equations to make them linear:

$$
\begin{aligned}
& 0 \leq z_{\omega}^{u p} \leq \bar{\phi} \Xi^{(1)} \\
& s_{\omega}-(1-\bar{\phi}) \Xi^{(1)} \leq z_{\omega}^{u p} \leq s_{\omega}
\end{aligned}
$$

The $\operatorname{Max}$ operator, $u_{\omega}^{+}=\operatorname{Max}\left(\Delta_{\omega}^{+}-\bar{\alpha}, 0\right)$, in the probabilistic constraint can be linearized as given by:

$$
\begin{aligned}
& 0 \leq u_{\omega}^{+} \leq b_{\omega}^{1} \Xi^{(1)} \\
& \Delta_{\omega}^{+}-\bar{\alpha} \leq u_{\omega}^{+} \leq \Delta_{\omega}^{+}-\bar{\alpha}+\left(1-b_{\omega}^{1}\right) \Xi^{(1)}
\end{aligned}
$$

Again, the other $\operatorname{Max}$ operator, $u_{\omega}^{-}=\operatorname{Max}\left(\Delta_{\omega}^{-}-\underline{\alpha}, 0\right)$, can be replaced by:

$$
\begin{aligned}
& 0 \leq u_{\omega}^{-} \leq b_{\omega}^{2} \Xi^{(1)} \\
& \Delta_{\omega}^{-}-\underline{\alpha} \leq u_{\omega}^{-} \leq \Delta_{\omega}^{-}-\underline{\alpha}+\left(1-b_{\omega}^{2}\right) \Xi^{(1)}
\end{aligned}
$$

\section{B. Linearizations related to SIP-SR model}

The non-linearity introduced because of up-regulation price and total negative deviation in the SIP-SR model is given by $c_{\omega}^{d n}=\operatorname{Max}\left(\lambda_{i}^{u p} N_{i, \omega}^{u p}\right)$, in order to linearize it, it can be replaced with the following equations:

$$
\begin{aligned}
& -r_{i \omega} \Xi^{(1)} \leq c_{\omega}^{u p}-\lambda_{i}^{u p} N_{i, \omega}^{u p} \leq r_{i \omega} \Xi^{(1)} \\
& \sum_{i} r_{i \omega}=\mathcal{G}-1
\end{aligned}
$$

where $r_{i \omega}$ is a binary variable.

For the 'If' condition, $\mathbb{I}\left(\sum_{i} \phi_{i}^{u p} g_{i}^{u p}<\sum_{i} \phi_{i}^{d n} g_{i}^{d n}\right)$, the below equations can be used:

$$
\begin{aligned}
\sum_{i} \phi_{i}^{u p} g_{i}^{u p} & \geq \sum_{i} \phi_{i}^{d n} g_{i}^{d n}-\Xi^{(1)}(1-\underline{\phi})+\epsilon \\
\sum_{i} \phi_{i}^{u p} g_{i}^{u p} & \leq \sum_{i} \phi_{i}^{d n} g_{i}^{d n}+\Xi^{(1)} \underline{\phi}-\epsilon
\end{aligned}
$$

The non-linear expression, $k_{i}^{d n}=\phi \phi_{i}^{d n}$, can be linearized as $\phi+\phi_{i}^{d n}-1 \leq k_{i}^{d n} \leq \phi$ and $k_{i}^{d n} \leq \phi_{i}^{d n}$

From the objective function 26, $t_{\omega}^{u p}=\Delta_{\omega}^{+} \bar{\phi}$ can be replaced by the following equations:

$$
\begin{aligned}
& 0 \leq t_{\omega}^{u p} \leq \bar{\phi} \Xi^{(1)} \\
& \Delta_{\omega}^{+}-(1-\bar{\phi}) \Xi^{(1)} \leq t_{\omega}^{u p} \leq \Delta_{\omega}^{+}
\end{aligned}
$$

Another non-linear term, $h^{+}=\Delta_{\omega}^{+} \phi$, from 26 can be written as:

$$
\begin{aligned}
& 0 \leq h^{+} \leq \phi \Xi^{(1)} \\
& \Delta_{\omega}^{+}-(1-\phi) \Xi^{(1)} \leq h^{+} \leq \Delta_{\omega}^{+}
\end{aligned}
$$

On similar lines, the term $\Delta_{\omega}^{-} \phi$, in $\sqrt{26}$, can be denoted by $t_{\omega}^{d n}$ and replaced with:

$$
\begin{aligned}
& 0 \leq t_{\omega}^{d n} \leq \underline{\phi} \Xi^{(1)} \\
& \Delta_{\omega}^{-}-(1-\underline{\phi}) \Xi^{(1)} \leq t_{\omega}^{d n} \leq \Delta_{\omega}^{-}
\end{aligned}
$$

Finally, the non-linearity introduced in 26 , by $h^{-}=\Delta_{\omega}^{-} \phi$ can be tackled by replacing it with the following equations:

$$
\begin{aligned}
& 0 \leq h^{-} \leq \phi \Xi^{(1)} \\
& \Delta_{\omega}^{-}-(1-\phi) \Xi^{(1)} \leq h^{-} \leq \Delta_{\omega}^{-}
\end{aligned}
$$

Please note that, in our case, the same value of $\Xi$ worked for all the linearization equations. But it can also be different corresponding to each specific linearization, even though we have written $\Xi^{(1)}$ throughout the Appendix section. 